\title{
Necesidades profesionales del profesorado especialista de Música de los centros de Primaria de Castilla La Mancha
}

\author{
Professional needs of music education teachers in Primary schools in Castilla La \\ Mancha
}

\author{
Narciso José López García \\ narcisojose.lopez@uclm.es \\ Departamento de Didáctica de la Expresión Musical, Plástica y Corporal
} Facultad de Educación de Albacete. Universidad de Castilla-La Mancha

doi: 10.7203/LEEME.39.9904

Recibido: 21-2-17 Aceptado: 23-5-17. Contacto y correspondencia: Narciso José López García Facultad de Educación de Albacete. Universidad de Castilla La Mancha. Plaza de la Universidad, 3. 02071 Albacete

\section{Resumen}

La nueva estructura curricular establecida por la LOMCE ha relegado a la Educación Musical al bloque de asignaturas específicas (voluntarias), veinticinco años después de haber sido incorporada como materia obligatoria en los currículos de Primaria. Esta modificación en la estructura de nuestro sistema educativo y las realizadas por las Leyes de Educación puestas en marcha con la LOGSE y la LOMCE, se han producido sin contar con la opinión de los protagonistas en los procesos educativos: los propios docentes. Esta investigación recoge la opinión y la percepción de los maestros especialistas de música de Castilla-La Mancha, Comunidad Autónoma en la que ejercen su labor como profesionales de la Educación Musical, sobre el estado actual de esta disciplina en las escuelas de Primaria, así como las necesidades y dificultades profesionales a las que se enfrentan día a día en su labor de aula. Para ello, se plantearon una serie de interrogantes y un objetivo general, que quedó concretado en ocho objetivos específicos, a los que se les ha intentado dar respuesta. Nuestro método de trabajo, el estudio descriptivo, se ha valido tanto de herramientas cuantitativas (dos cuestionarios electrónicos de elaboración propia) como cualitativas (la Técnica Delphi Online) para la recogida, análisis e interpretación de datos, por lo que el enfoque metodológico utilizado queda definido dentro de la denominada Metodología Mixta. Estas herramientas nos han servido para conocer los recursos tanto materiales como humanos destinados a la enseñanza de la música en los centros de Educación Primaria castellano-manchegos. Finalmente, se exponen las conclusiones, limitaciones y prospectivas de futuro y se abren nuevas vías de investigación con las que se pretende solventar la escasez de estudios similares en nuestra región.

Palabras clave: Educación Musical, Educación Primaria, Necesidades del profesorado, Desarrollo profesional.

\section{Abstract}

The new curriculum established by LOMCE, acronym of the last educational reform in Spain, has relegated Music Education into the category of elective subject, twenty-five years after being included as a compulsory subject in the curriculum of primary education. This change in the structure of our educational system and those made by the Acts of Education launched by both LOGSE and LOMCE have occurred without taking into account the opinion of the main actors in education: teachers themselves. Our research reflects the opinion and perception of music teachers in Castilla-La Mancha, the Autonomous Region in which we carried out our work as professionals in Music Education. We studied the current state of this discipline in primary schools as well as the professional needs and difficulties that teachers face every day in class. In order to pursue this line of research, we asked a series of questions in order to reach a general objective, through eight specific objectives. We followed as a method the case study using both quantitative (two self-created surveys) and qualitative (Delphi Method) approaches (mixed methods) to both collect and to analyze data. These strategies have served us to know the resources both, material and human, destined to the teaching of music education in schools of Primary Education in Castilla-La Mancha. Finally, conclusions, limitations, and prospects for the future are presented as well as a showing of new fields of research to overcome the lack of similar studies in our region.

\section{Key words: Music education, Primary education, Teachers' needs, Professional development.}

NARCISO JOSÉ LÓPEZ GARCíA. THE CONTENT OF THIS ARTICLE IS THE SOLE RESPONSIBILITY OF THE AUTHORS. THE REVISTA ELECTRÓNICA DE LEEME AND UNIVERSITAT DE VALËNCIA ARE NOT LABLE FOR ANY LEGALACTONG THAT MAY ARE IS MÚSICA EN LA EDUCACIÓN-HTTP://MUSICA.REDIRIS.ES.ISSN: 1575-9563 EDITORES: UNIVERSIDAD DE VALENCIA Y JESÚS TEJADA GIMÉNEZ. VISIBILIDAD DE ESTA REVISTA: EBSCO, CINDOC (CESIC), CITEFACTOR, COPAC, DIALNET, DICE (CSIC), DOAJ, E-REVISTAS (CSIC), EBSCO PREMIER, ERIH+, GALE CENGAGE LEARNING, IN-RECS, IRESIE, LATINDEX, MIAR, OCLC WORLDCAT, RESH, REDIB, RILM CORE JOURNALS, SUDOC, ULRICHS, ZEITSCHRIFTDATENBANK, ESTA REVISTA ESTÁ PUBLICADA CON EL APOYO INSTITUCIONAL DE REDIRIS-CONSEJO SUPERIOR DE INVESTIGACIONES CIENTIFICAS Y ES DE ACCESO LIBRE. 


\section{Introducción}

En el curso escolar 2015/2016, se cumplieron veinticinco años de la incorporación de la Educación Musical como materia obligatoria en los currículos oficiales de Primaria de nuestro sistema educativo. En este período, hemos vivido la redacción, aprobación, puesta en marcha y derogación de varias Leyes de Educación, en las que la música en esta etapa educativa no ha terminado de ocupar un lugar equiparable al resto de Áreas Curriculares. De hecho, la Ley vigente (LOMCE), ha desplazado la enseñanza de la música al bloque de asignaturas específicas, perdiendo su condición de materia obligatoria.

Por otro lado, Carbajo (2009), Domínguez (2014) y López García (2015), entre otros, señalan que las nuevas titulaciones de Grado en Maestro de Primaria surgidas del denominado Plan Bolonia, han apostado por la preparación de docentes con un perfil generalista, con opción a elegir una Mención Cualificadora en Educación Musical que nada tiene que ver con las Especialidades surgidas de la aplicación de la LOGSE.

Esta nueva situación ha ocasionado un escenario educativo-musical muy distinto que, sin duda, merece una reflexión seria. Desde esta perspectiva, es importante, y obligatorio, conocer la opinión de los docentes encargados de impartir esta materia en las escuelas.

Este trabajo se ha centrado en el profesorado de Castilla-La Mancha, Comunidad Autónoma en la que se desarrolla nuestra labor como profesionales de la Educación Musical, y de la que se disponen escasos datos sobre la situación actual de los maestros de música en Primaria, sus puntos de vista sobre su quehacer diario en el aula, el material del que disponen para llevar a cabo su labor educativa, el grado de idoneidad de este material, el estado de las aulas de música o la opinión de la comunidad educativa a la que pertenecen sobre la enseñanza de la música en la escuela, entre otros asuntos. Todo desde una aproximación a la práctica docente de este colectivo, lo que nos ha permitido localizar las dificultades reales con las que se enfrentan día a día estos maestros.

En este sentido y, una vez revisada la bibliografía relacionada con este campo de investigación, se ha descubierto que, si bien existen algunas investigaciones relacionadas con la didáctica de diferentes disciplinas escolares y con las necesidades profesionales de otros especialistas y del profesorado generalista, como los trabajos de Gil, Perona, Andrés y Hernández (2001), Serrano, Lera y Contreras (2007), De Moya (2006) o De Moya, Bravo y García (2008), estamos ante un tema de estudio novedoso en esta Comunidad Autónoma, ya que no existen trabajos similares que recojan la opinión y percepción de los maestros de música en activo sobre la situación actual de la enseñanza de la música en las escuelas castellanomanchegas. Esto ha comportado la obligación de ser extremadamente rigurosos a la hora de seleccionar los aspectos sobre los que se ha recogido información y la necesidad y conveniencia

NARCISO JOSÉ LÓPEZ GARCÍA. THE CONTENT OF THIS ARTICLE IS THE SOLE RESPONSIBILITY OF THE AUTHORS. THE REVISTA ELECTRÓNICA DE LEEME AND UNIVERSITAT DE VALĖNCIA ARE NOT LIABLE FOR ANY LEGAL ACTIONS THAT MAY ARISE INVOLVING THE ARTICLE'S CONTENT. REVISTA ELECTRÓNICA DE LEEME - LISTA ELECTRÓNICA EUROPEA DE MÚSICA EN LA EDUCACIÓN-HTTP://MUSICA.REDIRIS.ES.ISSN: 1575-9563 EDITORES: UNIVERSIDAD DE VALENCIA Y JESÚS TEJADA GIMÉNEZ. VISIBILIDAD DE ESTA REVISTA: EBSCO, CINDOC (CESIC), CITEFACTOR, COPAC, DIALNET, DICE (CSIC), DOAJ, E-REVISTAS (CSIC), EBSCO PREMIER, ERIH+, GALE CENGAGE LEARNING, IN-RECS, IRESIE, LATINDEX, MIAR, OCLC WORLDCAT, RESH, REDIB, RILM CORE JOURNALS, SUDOC, ULRICHS, ZEITSCHRIFTDATENBANK, ESTA REVISTA ESTÁ PUBLICADA CON EL APOYO INSTITUCIONAL DE REDIRISCCONSEJO SUPERIOR DE INVESTIGACIONES CIENTIFICAS Y ES DE ACCESO LIBRE. 
de los mismos para que se puedan convertir en verdaderos generadores de conocimiento científico.

\section{Planteamiento del problema}

La evolución histórica de la educación española nos ha mostrado que los grandes cambios en nuestro sistema educativo se han producido de espaldas a la realidad profesional (Monarca, Fernández-González y Piedrahita, 2016) cayendo, una y otra vez, en el error de diseñar nuevas normas sin contar con la opinión de los verdaderos protagonistas del sistema: los docentes que, en palabras de Domínguez (2014), se sienten abandonados por sus representantes políticos cuando acuden a ellos para defender esta materia.

Nuestro punto de partida surge, por tanto, de la necesidad de otorgar protagonismo a los maestros especialistas en Educación Musical de las escuelas de Primaria de Castilla-La Mancha ya que, como señala Carbajo (2009, p. 321), "cualquier intento de acercamiento a la realidad de un colectivo ha de basarse en la opinión de las personas que lo forman".

Partiendo de esta premisa, la finalidad última de este estudio se ha centrado en obtener una radiografía del estado real en el que se encuentra la Educación Musical en los colegios de nuestra región, cuáles son las necesidades profesionales de los docentes encargados de su magisterio y a qué tipo de obstáculos se enfrentan día a día en el aula que puedan estar dificultando su labor. Este colectivo se ha convertido, por tanto, en pilar fundamental, agente protagonista del sistema educativo y "fuente primaria específica de información" sobre la realidad educativo-musical desarrollada en nuestras aulas.

Desde esta posición, esta investigación se ha propuesto conocer, entender e interpretar qué está pasando en torno a la enseñanza de la música en las escuelas castellano-manchegas de la mano de sus docentes, poseedores de un conocimiento exclusivo y fundamental a la hora de comprender qué sucede dentro del aula, lo que proporcionará un conocimiento profundo y real de su estructura y funcionamiento y nos descubrirá todos aquellos aspectos susceptibles de mejora (Morales, 2008).

\section{Interrogantes a resolver}

Haciendo nuestros los postulados de Latorre, Rincón y Arnal (2003), esta investigación se ha aproximado a la realidad de los hechos de estudio atendiendo a los siguientes interrogantes:

1. ¿Cuáles son las características profesionales generales de este colectivo docente?

2. ¿Cómo influyen estas características profesionales en su labor docente?

NARCISO JOSÉ LÓPEZ GARCÍA. THE CONTENT OF THIS ARTICLE IS THE SOLE RESPONSIBILITY OF THE AUTHORS. THE REVISTA ELECTRÓNICA DE LEEME AND UNIVERSITAT DE VALĖNCIA ARE NOT LIABLE FOR ANY LEGAL ACTIONS THAT MAY ARISE INVOLVING THE ARTICLE'S CONTENT. REVISTA ELECTRÓNICA DE LEEME -LISTA ELECTRÓNICA EUROPEA DE MÚSICA EN LA EDUCACIÓN-HTTP://MUSICA.REDIRIS. ES.ISSN: 1575-9563 EDITORES: UNIVERSIDAD DE VALENCIA Y JESÚS TEJADA GIMÉNEZ. VISIBILIDAD DE ESTA REVISTA: EBSCO, CINDOC (CESIC), CITEFACTOR, COPAC, DIALNET, DICE (CSIC), DOAJ, E-REVISTAS (CSIC), EBSCO PREMIER, ERIH+, GALE CENGAGE LEARNING, IN-RECS, IRESIE, LATINDEX, MIAR, OCLC WORLDCAT, RESH, REDIB, RILM CORE JOURNALS, SUDOC, ULRICHS,
SUPERIOR DE INVESTIGACIONES CIENTIFICAS Y ES DE ACCESO LIBRE. 
3. ¿Cuál es el grado de conocimiento de este colectivo docente sobre las teorías relacionadas con la Educación Musical más importantes y sobre los nuevos currículos basados en el desarrollo de competencias?

4. ¿Cuál es la visión del especialista de música sobre la legislación educativo-musical y su relación con la realidad vivida en los centros con respecto a la enseñanza de la música?

5. ¿Cómo influye el contexto educativo general actual en los procesos de enseñanza aprendizaje de la música en las escuelas castellano - manchegas?

6. ¿Qué dificultades recurrentes aparecen en este colectivo docente tras varios cambios legislativos y cuáles son las necesidades y demandas que más se repiten entre estos profesionales?

7. ¿Qué tipo de actividades de formación permanente desarrollan y cómo valoran esta formación?

\section{Objetivos}

Una vez definidos los interrogantes a resolver, nos planteamos el siguiente objetivo general:

Conocer las características profesionales generales de los maestros especialistas de música de los centros de Primaria de Castilla-La Mancha y detectar, recoger y analizar las necesidades de este colectivo en el ejercicio de su labor docente.

Este objetivo general se concreta en los siguientes objetivos específicos:

1. Describir las características generales del colectivo de docentes especialistas en Educación Musical de los centros de Primaria de Castilla-La Mancha.

2. Conocer su autopercepción sobre su propio grado de preparación en competencias y capacidades educativo-musicales.

3. Analizar las características profesionales más relevantes de este profesorado.

4. Realizar un sondeo sobre los recursos materiales de los que disponen para llevar a cabo su labor docente, su estado y el grado de adecuación de los mismos.

5. Conocer y analizar sus percepciones sobre la opinión que los componentes de la comunidad educativa a la que pertenecen (las familias y el resto de docentes) tienen de la Educación Musical en la escuela.

6. Detectar los problemas más comunes de este colectivo docente, si los hay, en su práctica educativa dentro del aula.

7. Analizar el grado de implicación de este profesorado ante los diferentes modelos de formación permanente que tiene a su disposición.

8. Conocer sus percepciones sobre la situación de la Educación Musical en la legislación educativa actual.

NARCISO JOSÉ LÓPEZ GARCÍA. THE CONTENT OF THIS ARTICLE IS THE SOLE RESPONSIBILITY OF THE AUTHORS. THE REVISTA ELECTRÓNICA DE LEEME AND UNIVERSITAT DE VALÈNCIA ARE NOT LABLE FOR ANY LEGAL ACTIONS THAT MAY ARISE INVOLVING THE ARTICLE'S CONTENT. REVISTA ELECTRÓNICA DE LEEME - LISTA ELECTRÓNICA EUROPEA DE MÚSICA EN LA EDUCACIÓN-HTTP:/ /MUSICA.REDIRIS.ES.ISSN: 1575-9563 EDITORES: UNIVERSIDAD DE VALENCIA Y JESÚS TEJADA GIMÉNEZ. VISIBILIDAD DE ESTA REVISTA: EBSCO, CINDOC (CESIC), CITEFACTOR, COPAC, DIALNET, DICE (CSIC), DOAJ, E-REVISTAS (CSIC), EBSCO PREMIER, ERIH+, GALE CENGAGE LEARNING, IN-RECS, IRESIE, LATINDEX, MIAR, OCLC WORLDCAT, RESH, REDIB, RILM CORE JOURNALS, SUDOC, ULRICHS, ZEITSCHRIFTDATENBANK, ESTA REVISTA ESTÁ PUBLICADA CON EL APOYO INSTITUCIONAL DE REDIRIS-CONSEJO SUPERIOR DE INVESTIGACIONES CIENTIFICAS Y ES DE ACCESO LIBRE. 


\section{Método}

Este trabajo, enmarcado dentro de la denominada investigación educativa, se ha llevado a cabo combinando los paradigmas cuantitativo y cualitativo sacando provecho, como dice Malbrán (2006), de ambas perspectivas y de la simbiosis paradigmática que existe en la actualidad. Hablamos, por lo tanto, de una Metodología Mixta ya que ha recogido, analizado y vinculado datos cuantitativos y cualitativos en el mismo estudio para dar respuesta a los planteamientos que nos hemos propuesto. Además, no se ha limitado a la simple recogida de datos de diferente naturaleza, sino que ha combinado la lógica inductiva con la deductiva a lo largo de todo el proceso investigador, abarcando el planteamiento del problema, el análisis de datos, la interpretación de los resultados obtenidos y el informe final (Castañer, Camerino y Anguera, 2013).

En este sentido, Castro y Godino (2011, p. 100) afirman que las Metodologías Mixtas "permiten comprender las actividades educativas en el contexto en que tienen lugar y al mismo tiempo aportar recomendaciones generalizables que apoyen la toma de decisiones en política educativa". Sin embargo, la cuantificación no ha supuesto ni el punto de inicio ni el objetivo último del estudio, por lo que no constituye su eje fundamental. No obstante, ha servido para reforzar algunos de los datos obtenidos y realizar sencillas fórmulas numéricas con las que complementar el análisis de calidad y fiabilidad del mismo.

El método de trabajo seleccionado, el estudio descriptivo, ha permitido descubrir e interpretar las particularidades y peculiaridades de la labor docente de los maestros de música en las aulas de las escuelas de Castilla-La Mancha (Sabariego, 2010). Asimismo, haciendo nuestros los parámetros de Álvarez y San Fabián (2012), este estudio descriptivo nos ha facultado para generalizar a partir de un conjunto de situaciones específicas con la intención de profundizar en ellas, a la vez que nos ha ofrecido descripciones ricas y densas con las que emitir juicios sobre la realidad objeto de estudio.

\section{Técnicas e instrumentos de recogida de datos}

El instrumento fundamental de recogida de datos ha sido un cuestionario electrónico (CE-01), de creación propia, con el que se ha recopilado la información necesaria para cumplir con el objetivo general de la investigación. Además, se ha utilizado un segundo cuestionario electrónico (CE-02), también de creación propia, ambos validados por un comité de expertos (validación basada en criterios de pertinencia, de adecuación y de contenido de los mismos) y sometidos a la prueba de validación de caso único. Por otro lado, se ha realizado la Técnica Delphi, que se explicará en los siguientes epígrafes, que ha servido en el proceso de análisis y verificación de la información obtenida mediante la triangulación de los datos compilados.

NARCISO JOSÉ LÓPEZ GARCÍA. THE CONTENT OF THIS ARTICLE IS THE SOLE RESPONSIBILITY OF THE AUTHORS. THE REVISTA ELECTRÓNICA DE LEEME AND UNIVERSITAT DE VALĖNCIA ARE NOT LIABLE FOR ANY LEGAL ACTIONS THAT MAY ARISE INVOLVING THE ARTICLE'S CONTENT. REVISTA ELECTRÓNICA DE LEEME -LISTA ELECTRÓNICA EUROPEA DE MÚSICA EN LA EDUCACIÓN-HTTP://MUSICA.REDIRIS.ES.ISSN: 1575-9563 EDITORES: UNIVERSIDAD DE VALENCIA Y JESÚS TEJADA GIMÉNEZ. VISIBILIDAD DE ESTA REVISTA: EBSCO, CINDOC (CESIC), CITEFACTOR, COPAC, DIALNET, DICE (CSIC), DOAJ, E-REVISTAS (CSIC), EBSCO PREMIER, ERIH+, GALE CENGAGE LEARNING, IN-RECS, IRESIE, LATINDEX, MIAR, OCLC WORLDCAT, RESH, REDIB, RILM CORE JOURNALS, SUDOC, ULRICHS, ZEITSCHRIFTDATENBANK, ESTA REVISTA ESTÁ PUBLICADA CON EL APOYO INSTITUCIONAL DE REDIRIS-CONSEJO SUPERIOR DE INVESTIGACIONES CIENTIFICAS Y ES DE ACCESO LIBRE. 


\subsection{El cuestionario electrónico CE-01}

Con este cuestionario se han recopilado todos aquellos aspectos relacionados con las opiniones, percepciones y necesidades profesionales de los especialistas de música castellanomanchegos. Para su elaboración, teniendo en cuenta la falta de trabajos similares en nuestra Comunidad Autónoma, recurrimos a la búsqueda de formularios y trabajos realizados en investigaciones análogas a la nuestra, llevadas a cabo en otras regiones españolas, especialmente los presentados por Carbajo (2008; 2009), Epelde (2005), Longueira (2011), Ocaña (2006) y Reyes (2005).

Este instrumento incluía los dos tipos básicos de preguntas: de identidad del sujeto y de contenido (Giráldez, 2006), todas ellas cerradas a excepción de la última respuesta que, al ser abierta, nos ha permitido recoger observaciones y sugerencias de los participantes.

El CE-01 pertenece a los cuestionarios del tipo referido a la norma, ya que todas las preguntas son válidas y correctas, por lo que los participantes han tenido que señalar la que mejor se ajustaba a su realidad diaria (Lozano y De la Fuente, 2009). Sobre los ítems que lo configuran, éstos son de respuestas dicotómicas, del tipo SÍ-NO, y de escalas Likert, del tipo 1Nunca, 2-Pocas veces, 3-A veces, 4-Muchas veces, 5-Siempre (escala ordinal de cinco puntos).

El CE-01 se ha estructurado en siete bloques de preguntas atendiendo a siete variables diferentes:

1. Primer bloque: Datos de identificación de sujetos y formación académica.

2. Segundo bloque: Datos generales sobre el centro de trabajo, la localidad en la que se encuentra y la situación administrativa de la plaza ocupada.

3. Tercer bloque: Datos generales sobre la práctica docente.

4. Cuarto bloque: Recursos materiales del aula y grado de adecuación de los mismos.

5. Quinto bloque: Percepción sobre la opinión que los docentes del centro tienen de la Educación Musical en Primaria.

6. Sexto bloque: Percepción sobre la opinión del resto de la comunidad educativa acerca de la Educación Musical en Primaria.

7. Séptimo bloque: Otros datos.

Con respecto a la población y a la muestra seleccionadas, optamos por utilizar un muestreo no probabilístico intencional (Cardona, 2002; Tójar y Matas, 2009), haciendo coincidir la población con la muestra.

En el momento de enviar el CE-01, nuestra Comunidad Autónoma contaba con 610 maestros de música de Primaria (según Orden de 31/01/2012, de la Consejería de Educación, Cultura y Deportes, por la que se publica la plantilla, la composición de unidades y otros datos

NARCISO JOSÉ LÓPEZ GARCÍA. THE CONTENT OF THIS ARTICLE IS THE SOLE RESPONSIBILITY OF THE AUTHORS. THE REVISTA ELECTRÓNICA DE LEEME AND UNIVERSITAT DE VALĖNCIA ARE NOT LIABLE FOR ANY LEGAL ACTIONS THAT MAY ARISE INVOLVING THE ARTICLE'S CONTENT. REVISTA ELECTRÓNICA DE LEEME -LISTA ELECTRÓNICA EUROPEA DE MÚSICA EN LA EDUCACIÓN-HTTP://MUSICA.REDIRIS.ES.ISSN: 1575-9563 EDITORES: UNIVERSIDAD DE VALENCIA Y JESÚS TEJADA GIMÉNEZ. VISIBILIDAD DE ESTA REVISTA: EBSCO, CINDOC (CESIC), CITEFACTOR, COPAC, DIALNET, DICE (CSIC), DOAJ, E-REVISTAS (CSIC), EBSCO PREMIER, ERIH+, GALE CENGAGE LEARNING, IN-RECS, IRESIE, LATINDEX, MIAR, OCLC WORLDCAT, RESH, REDIB, RILM CORE JOURNALS, SUDOC, ULRICHS, ZEITSCHRIFTDATENBANK, ESTA REVISTA ESTÁ PUBLICADA CON EL APOYO INSTITUCIONAL DE REDIRIS-CONSEJO SUPERIOR DE INVESTIGACIONES CIENTIFICAS Y ES DE ACCESO LIBRE. 
de determinados centros públicos de Educación Infantil y Primaria, Colegios Rurales Agrupados y Colegios de Educación Especial) ${ }^{1}$ Terminado el plazo de entrega de cuestionarios, el número de participantes ascendía a $242(\mathrm{n}=242)$, lo que hacía a la muestra representativa de la población (véase Tabla 1).

Por otro lado, esta herramienta fue sometida a diferentes pruebas de validez y fiabilidad con las que se comprobó el grado de idoneidad de la misma con respecto a los objetivos planteados en nuestra investigación. Una vez conocidos los coeficientes de dichas pruebas, pudimos corroborar que el CE-01 reunía las cualidades esenciales que se demandan a este tipo de instrumentos.

Tabla 1. Pruebas de validez y fiabilidad a las que fue sometido el cuestionario CE-01

\begin{tabular}{|c|c|}
\hline \multicolumn{2}{|c|}{ Instrumentos de validez y fiabilidad empleados } \\
\hline Validez & Fiabilidad \\
\hline Validación de caso único: & Poblaciones finitas: \\
\hline $\begin{array}{l}\text { Aplicación del cuestionario a una persona de confianza } \\
\text { con las mismas características que los destinatarios de la } \\
\text { prueba. } \\
\text { Valoración global: } 9 \text { '5 sobre } 10\end{array}$ & $\begin{array}{l}\text { El nivel de confianza obtenido se encuentra entre el } 95 \% \\
\text { y el } 96 \% \text {, cumpliendo con los criterios de } \\
\text { representatividad, validez y confiabilidad necesarios que } \\
\text { garantizan la generalización de los datos obtenidos. }\end{array}$ \\
\hline $\begin{array}{l}\text { Validación de expertos: } \\
\text { Llevada a cabo por } 20 \text { profesores de las Facultades de } \\
\text { Educación de distintas Universidades españolas. } \\
\text { Valoración global: 9'361 sobre } 10\end{array}$ & $\begin{array}{l}\text { Tamaños muestrales (paquete estadístico SPSS 17.0): } \\
\text { Aplicando las tablas de Krejcie y Morgan, para una } \\
\text { población } N=650 \text { personas, la muestra debe ser } n=242 \text {, } \\
\text { por lo que nuestra muestra cumple con los criterios } \\
\text { generales de representatividad. }\end{array}$ \\
\hline $\begin{array}{l}\text { Aplicación piloto: } \\
\text { Realizada por } 10 \text { docentes relacionados con la } \\
\text { Educación Musical en Primaria y en Secundaria. } \\
\text { Valoración global: 9’37 }\end{array}$ & $\begin{array}{l}\text { Alfa de Cronbach (paquete estadístico SPSS 17.0): } 0^{\prime} 848 \\
\text { El coeficiente obtenido se sitúa dentro de los criterios } \\
\text { generales de fiabilidad propuesto por los expertos. }\end{array}$ \\
\hline $\begin{array}{l}\text { Triangulación: } \\
\text { Mediante la Técnica Delphi y el Cuestionario CE- } 02\end{array}$ & $\begin{array}{l}\text { Dos mitades (paquete estadístico SPSS 17.0): } \\
\text { Coeficiente Spearman - Brown= 0'930 } \\
\text { Coeficiente de Guttman=0'929 } \\
\text { Ambos coeficientes cumplen los criterios de fiabilidad } \\
\text { aplicados. } \\
\text { W de Kendall (paquete estadístico SPSS 17.0): } \\
\text { El Coeficiente W de Kendall con el que se midió el grado } \\
\text { de acuerdo y el nivel de concordancia interjueces }=0{ }^{\prime} 833\end{array}$ \\
\hline
\end{tabular}

\footnotetext{
${ }^{1}$ El Decreto de plantillas recoge los puestos para maestros de música de los centros públicos. Para conocer el número de especialistas en los centros privados y concertados de nuestra región, fue necesario ponerse en contacto con los equipos directivos de los mismos, mediante llamadas telefónicas, los cuales facilitaron las cifras correspondientes al personal docente que impartía la asignatura de Educación Musical en dichos centros.

NARCISO JOSÉ LÓPEZ GARCÍA. THE CONTENT OF THIS ARTICLE IS THE SOLE RESPONSIBILITY OF THE AUTHORS. THE REVISTA ELECTRÓNICA DE LEEME AND UNIVERSITAT DE VALĖNCIA ARE NOT LIABLE FOR ANY LEGAL ACTIONS THAT MAY ARISE INVOLVING THE ARTICLE'S CONTENT. REVISTA ELECTRÓNICA DE LEEME - LISTA ELECTRÓNICA EUROPEA DE MÚSICA EN LA EDUCACIÓN-HTTP://MUSICA.REDIRIS.ES.ISSN: 1575-9563 EDITORES: UNIVERSIDAD DE VALENCIA Y JESÚS TEJADA GIMÉNEZ. VISIBILIDAD DE ESTA REVISTA: EBSCOO, CINDOC (CESIC), CITEFACTOR, COPAC, DIALNET, DICE (CSIC), DOAJ, E-REVISTAS (CSIC), EBSCO PREMIER, ERIH+, GALE CENGAGE LEARNING, IN-RECS, IRESIE, LATINDEX, MIAR, OCLC WORLDCAT, RESH, REDIB, RILM CORE JOURNALS, SUDOC, ULRICHS, ZEITSCHRIFTDATENBANK, ESTA REVISTA ESTÁ PUBLICADA CON EL APOYO INSTITUCIONAL DE REDIRIS-CONSEJO SUPERIOR DE INVESTIGACIONES CIENTIFICAS Y ES DE ACCESO LIBRE.
} 
LISTA ELECTRÓNICA EUROPEA DE MÚSICA EN LA EDUCACIÓN

\subsection{El proceso de triangulación}

Atendiendo a las tipologías definidas por Rodríguez Ruiz (2005), el tipo utilizado en nuestro estudio encaja con la triangulación metodológica intermétodos simultánea, en la que se han aplicado métodos cualitativos y cuantitativos al mismo tiempo buscando, de esta manera, analizar un mismo fenómeno a través de diversos acercamientos.

Las estrategias llevadas a cabo para triangular los datos obtenidos con el CE-01 han sido: la Técnica Delphi (técnica cualitativa) y un segundo cuestionario electrónico (técnica cuantitativa), al que hemos denominado CE-02.

\subsection{La Técnica Delphi}

La Técnica Delphi tiene por objeto "conocer la opinión de un grupo de personas en relación a un problema, sin que éstas se reúnan físicamente" (Campoy y Gomes, 2009, p. 286), siendo su objetivo fundamental lograr un consenso fiable entre las opiniones de un grupo experto en el tema o fenómeno a estudiar.

En nuestro caso, utilizamos la variante Delphi Online agilizando, de esta forma, el proceso de envío y recepción de información entre investigador y expertos.

Con respecto a la muestra siguiendo, de nuevo, las indicaciones de Campoy y Gomes (2009), decidimos seleccionar al azar a 20 especialistas de música de los 242 que habían contestado al CE-01, 4 sujetos por provincia asegurándonos, así, el grado de conocimiento de los mismos sobre el problema de estudio y su grado de motivación e implicación personal ante el tema de estudio.

Tabla 2. Fases de realización de la Técnica Delphi Técnica Delphi

Determinación del problema a debatir y formulación de las preguntas

Selección de la muestra

Envío de instrucciones, del calendario orientativo y del primer bloque de preguntas

Recogida y análisis de respuestas, configuración y envío del segundo bloque de preguntas

Recogida y análisis de respuestas, configuración y envío del tercer bloque de preguntas

Conclusiones e informe final

Envío del informe a los participantes junto con los agradecimientos por su colaboración

NARCISO JOSÉ LÓPEZ GARCIAA. THE CONTENT OF THIS ARTICLE IS THE SOLE RESPONSIBILITY OF THE AUTHORS. THE REVISTA ELECTRÓNICA DE LEEME AND UNIVERSITAT DE 法 MÚSICA EN LA EDUCACIÓN-HTTP.//MUSICA REDIRIS.ES.ISSN: 1575-9563 EDITORES" UNIVERSIDAD DE VALENCIA Y JESÚS TEJADA GIMÉNEZ. VISIBILIDAD DE ESTA REVISTA: EBSCO, CINDOC (CESIC), CITEFACTOR, COPAC, DIALNET, DICE (CSIC), DOAJ, E-REVISTAS (CSIC), EBSCO PREMIER, ERIH+, GALE CENGAGE LEARNING, IN-RECS, IRESIE, LATINDEX, MIAR, OCLC WORLDCAT, RESH, REDIB, RILM CORE JOURNALS, SUDOC, ULRICHS, ZEITSCHRIFTDATENBANK, ESTA REVISTA ESTÁ PUBLICADA CON EL APOYO INSTITUCIONAL DE REDIRIS-CONSEJO SUPERIOR DE INVESTIGACIONES CIENTIFICAS Y ES DE ACCESO LIBRE. 


\subsection{El cuestionario electrónico CE-02}

Diseñado utilizando la plataforma Google Drive, el cuestionario CE-02 se remitió al profesorado de los Departamentos de Didáctica de la Expresión Musical de las cuatro Facultades de Educación de la Universidad de Castilla-La Mancha.

La estructura de esta prueba recogía las ideas principales del CE-01, concretadas en 12 preguntas cerradas a responder según el tipo de respuesta de escala de Likert, y una última pregunta abierta con la que este profesorado pudo expresar su opinión sobre el estado actual de la Educación Musical en Primaria.

Las variables de estudio propuestas en el CE-02 fueron las siguientes:

1. Valoración del hecho de que un buen número de especialistas ejerzan labores de tutor.

2. Implicación de las administraciones locales en la programación de eventos musicales para escolares.

3. Valoración de los equipamientos básicos de las aulas de música.

4. Valoración de los equipamientos TIC de las aulas de música.

5. Valoración del mobiliario básico de las aulas de música.

6. Percepción sobre la opinión de la comunidad educativa hacia la Educación Musical en Primaria.

7. Percepción sobre la opinión del resto de docentes sobre la Educación Musical en Primaria.

8. Impulso de las leyes de educación a la Educación Musical.

9. Necesidad de establecer la enseñanza obligatoria de la música en la etapa de Educación Infantil.

10. Los puestos con carácter itinerante o compartido y su influencia en el desarrollo de los procesos de enseñanza-aprendizaje de la música.

\section{Resultados}

Los datos recogidos se presentan estructurados en tres grandes apartados: en el primero de ellos, se exponen los resultados acopiados mediante el CE-01; en el segundo se exponen los datos obtenidos mediante la Técnica Delphi; y, por último, se presentan los datos del CE-02.

En relación a la exposición de resultados, y conociendo que en otros trabajos de corte similar el agrupamiento de los mismos suele llevarse a cabo de acuerdo a las variables de estudio, se ha creído conveniente utilizar este formato buscando, con ello, una lectura más cómoda y ágil de los mismos. NARCISO JOSÉ LÓPEZ GARCía. THE CONTENT OF THIS ARTICLE IS THE SOLE RESPONSIBILITY OF THE AUTHORS. THE REVISTA ELECTRÓNICA DE LEEME AND UNIVERSITAT DE
VALĖNCIA ARE NOT LIABLE FOR ANY LEGAL ACTIONS THAT MAY ARISE INVOLVING THE ARTICLE'S CONTENT. REVISTA ELECTRÓNICA DE LEEME - LISTA ELECTRÓNICA EUROPEA DE MÚSICA EN LA EDUCACIÓN-HTTP://MUSICA.REDIRIS.ES.ISSN: 1575-9563 EDITORES: UNIVERSIDAD DE VALENCIA Y JESÚS TEJADA GIMÉNEZ. VISIBILIDAD DE ESTA REVISTA: EBSCO, CINDOC (CESIC), CITEFACTOR, COPAC, DIALNET, DICE (CSIC), DOAJ, E-REVISTAS (CSIC), EBSCO PREMIER, ERIH+, GALE CENGAGE LEARNING, IN-RECS, IRESIE, LATINDEX, MIAR, OCLC WORLDCAT, RESH, REDIB, RILM CORE JOURNALS, SUDOC, ULRICHS, ZEITSCHRIFTDATENBANK, ESTA REVISTA ESTÁ PUBLICADA CON EL APOYO INSTITUCIONAL DE REDIRISCCONSEJO SUPERIOR DE INVESTIGACIONES CIENTIFICAS Y ES DE ACCESO LIBRE. 


\subsection{El cuestionario CE-01: datos relevantes}

En Castilla-La Mancha contamos con 770 centros de Educación Primaria, de los cuales 657 son públicos, 107 son concertados y 6 son privados. El grupo mayoritario de participantes se encuentra situado en la franja etaria correspondiente a los 31-45 años (164 personas), ha cursado sus estudios en la Universidad de Castilla-La Mancha (205 personas) y su experiencia docente como especialistas de música no supera los diez años (146 personas).

Con respecto a su situación administrativa, 158 sujetos (65\%) son funcionarios de carrera, y sobre la forma de acceso a la docencia como especialistas de música, 176 personas (73\%) lo hicieron con la titulación correspondiente a la Especialidad de Educación Musical.El porcentaje de maestros con estudios musicales oficiales alcanza el 82\% (197 participantes), de los que 195 ya habían cursado parte de estos estudios al comienzo de la carrera de maestro.

Preguntados por sus labores en el centro, sólo 61 sujetos, de los 242 participantes, ejercen labores de tutor además de las labores correspondientes como especialistas de música y, en su gran mayoría, son docentes de centros privados o concertados.

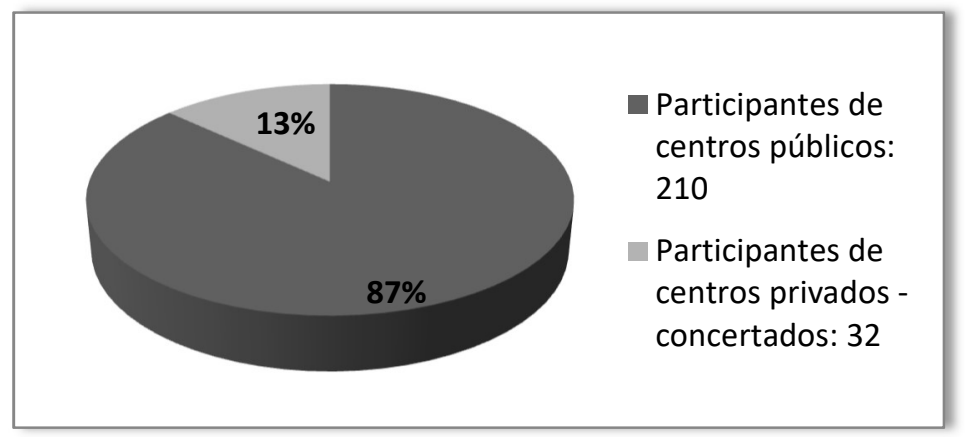

Figura 1. Número de encuestados atendiendo a la titularidad del centro en el que desempeñan su labor docente

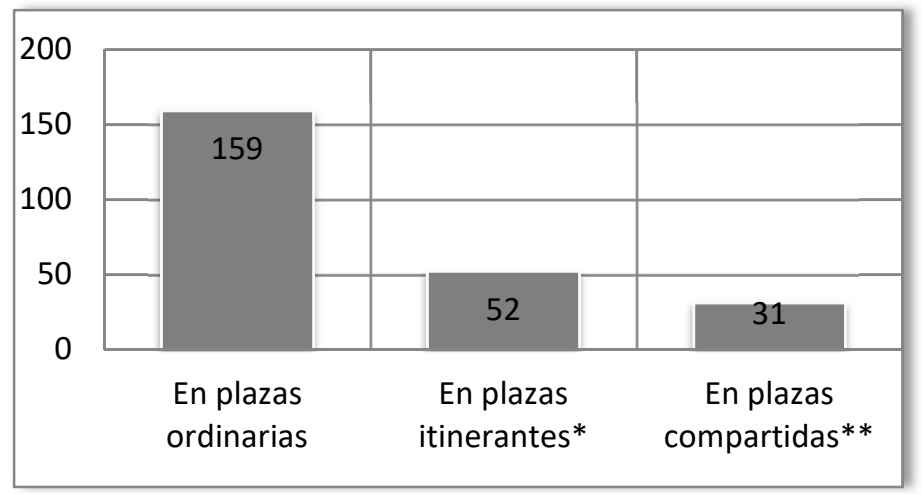

Figura 2. Número de encuestados atendiendo al perfil de la plaza en la que desempeñan su labor docente $(*$ Varios centros de distintas localidades; ** Varios centros de la misma localidad)

NARCISO JOSÉ LÓPEZ GARCÍA. THE CONTENT OF THIS ARTICLE IS THE SOLE RESPONSIBILITY OF THE AUTHORS. THE REVISTA ELECTRÓNICA DE LEEME AND UNIVERSITAT DE VALËNCIA ARE NOT LIABLE FOR ANY CONTENT OFNS THAT MAY ARISE INVOLVING THE ARTICLE'S CONTENT. REVISTA ELECTRÓNICA DE LEEME - LISTA ELECTRÓNICA EUROPEA DE MÚSICA EN LA EDUCACIÓN-HTTP://MUSICA.REDIRIS.ES.ISSN: 1575-9563 EDITORES: UNIVERSIDAD DE VALENCIA Y JESÚS TEJADA GIMÉNEZ. VISIBILIDAD DE ESTA REVISTA: EBSCO, CINDOC (CESIC), CITEFACTOR, COPAC, DIALNET, DICE (CSIC), DOAJ, E-REVISTAS (CSIC), EBSCO PREMIER, ERIH+, GALE CENGAGE LEARNING, IN-RECS, IRESIE, LATINDEX, MIAR, OCLC WORLDCAT, RESH, REDIB, RILM CORE JOURNALS, SUDOC, ULRICHS, ZEITSCHRIFTDATENBANK, ESTA REVISTA ESTÁ PUBLICADA CON EL APOYO INSTITUCIONAL DE REDIRIS-CONSEJO SUPERIOR DE INVESTIGACIONES CIENTIFICAS Y ES DE ACCESO LIBRE. 
Sobre el tipo de destino de los maestros que han realizado la encuesta, 168 definitivos, mientras que 53 cubren una vacante o realizan una sustitución y 13 ocupan otro tipo de destino. Existe una gran estabilidad en las plantillas de especialistas de música; en el momento de realizar la encuesta, 176 maestros afirmaban no tener intención de cambiar de destino. Sobre este asunto, diferentes expertos (Cardona, 2008; Thode, 1992; Tiana, 1993) sostienen que las condiciones laborales y la situación de inestabilidad del profesorado interino pueden incidir negativamente en los procesos de enseñanza-aprendizaje. A su vez, Cardona (2008) señala que una plantilla estable estará mejor preparada para afrontar con éxito todos aquellos retos propios de la práctica docente.

Los participantes nos han informado de que las localidades castellano-manchegas programan muy pocos eventos didáctico-musicales para escolares y solo en los municipios mayores de 10.000 habitantes encontramos unos porcentajes que, si bien siguen siendo muy bajos, superan levemente los índices generales. Este dato coincide con la información proporcionada por Salazar (2009), quien señala que la escasa oferta de espectáculos de este tipo por parte de las administraciones educativas (regionales, provinciales o locales) se ve reflejada en el reducido número de músicos y artistas que se dedican a este género, complicando enormemente la tarea de acercar el hecho musical al público escolar. Además, la administración regional encargada del circuito cultural y musical (Red de Teatros de Castilla-La Mancha), no contempla ayudas ni condiciones especiales para estas compañías, obligando a los municipios a costear el 100\% de los gastos de contratación de las mismas. Esto ha hecho que, en los últimos años, diferentes agrupaciones de toda España ofrezcan montajes didáctico-musicales directamente a los centros educativos castellano-manchegos, sin embargo, las dificultades económicas de éstos no están permitiendo su contratación.

Tabla 3. Opinión de los docentes sobre el $n^{\circ}$ de eventos musicales destinados a escolares

\begin{tabular}{|ccc|}
\hline \multicolumn{2}{|c|}{$\begin{array}{c}\text { Eventos programados y costeados por las } \\
\text { administraciones educativas }\end{array}$} \\
\hline ESCALA & $\mathrm{N}^{\circ}$ PARTICIPANTES & $\%$ \\
\hline Nada/Ninguno & 35 & 14 \\
Muy Pocos & 108 & 45 \\
Pocos/Algunos & 72 & 30 \\
Bastantes & 24 & 10 \\
Muchos & 3 & 1 \\
\hline Total & 242 & 100 \\
\hline
\end{tabular}

\begin{tabular}{|ccc|}
\hline \multicolumn{3}{|c|}{$\begin{array}{c}\text { Eventos programados y costeados por los centros } \\
\text { educativos }\end{array}$} \\
\hline ESCALA & $\mathrm{N}^{\circ}$ PARTICIPANTES & $\%$ \\
\hline Nada/Ninguno & 117 & 48 \\
Muy Pocos & 98 & 40 \\
Pocos/Algunos & 21 & 9 \\
Bastantes & 5 & 2 \\
Muchos & 3 & 1 \\
\hline Total & 242 & 100 \\
\hline
\end{tabular}

Existe un alto porcentaje de docentes (46\%) que no conoce a fondo la nueva estructuración curricular por competencias de la Educación Primaria. Este hecho, de especial interés y gravedad, coincide con las conclusiones alcanzadas por Monarca, Fernández-González NARCISO JOSÉ LÓPEZ GARCía. THE CONTENT OF THIS ARTICLE IS THE SOLE RESPONSIBILITY OF THE AUTHORS. THE REVISTA ELECTRÓNICA DE LEEME AND UNIVERSITAT DE VALĖNCIA ARE NOT LIABLE FOR ANY LEGAL ACTIONS THAT MAY ARISE INVOLVING THE ARTICLE'S CONTENT. REVISTA ELECTRÓNICA DE LEEME - LISTA ELECTRÓNICA EUROPEA DE MÚSICA EN LA EDUCACIÓN-HTTP://MUSICA.REDIRIS.ES.ISSN: 1575-9563 EDITORES: UNIVERSIDAD DE VALENCIA Y JESÚS TEJADA GIMÉNEZ. VISIBILIDAD DE ESTA REVISTA: EBSCO, CINDOC (CESIC), CITEFACTOR, COPAC, DIALNET, DICE (CSIC), DOAJ, E-REVISTAS (CSIC), EBSCO PREMIER, ERIH+, GALE CENGAGE LEARNING, IN-RECS, IRESIE, LATINDEX, MIAR, OCLC WORLDCAT, RESH, REDIB, RILM CORE JOURNALS, SUDOC, ULRICHS, ZEITSCHRIFTDATENBANK, ESTA REVISTA ESTÁ PUBLICADA CON EL APOYO INSTITUCIONAL DE REDIRIS-CONSEJO SUPERIOR DE INVESTIGACIONES CIENTIFICAS Y ES DE ACCESO LIBRE. 
y Piedrahita (2016), Sauras (2016) y Vázquez-Cano (2016), los cuales señalan la complejidad a la que se enfrenta el profesorado en los procesos de planificación, coordinación, desarrollo y evaluación por competencias. Además, Vázquez-Cano (2016) hace hincapié en la escasa formación relacionada con este tema que se ha ofrecido al colectivo docente.

Por otro lado, los participantes afirman conocer y utilizar las teorías tradicionales sobre Educación Musical, sin embargo, las nuevas metodologías relacionadas con la creatividad, la música contemporánea y las TIC son menos conocidas y, por tanto, menos utilizadas en el aula. De hecho, si bien señalan disponer de recursos suficientes y adecuados, el uso de las TIC como herramienta de apoyo a las estrategias metodológicas no es frecuente. El 44\% de los encuestados ha contestado que prácticamente no las utiliza. Estos datos se asemejan a los mostrados por Mominó, Sigalés y Meneses (2008) y Hernández (2011). Por otro lado, coincidiendo con los resultados obtenidos por Guerrero (2014) y Colás y Hernández (2014), se pone de manifiesto la falta de formación del profesorado, ya sea por el poco interés que despierta el uso de las TIC en los procesos de enseñanza-aprendizaje o por la escasez o falta de calidad de programas de formación en TIC desarrollados por las administraciones educativas.

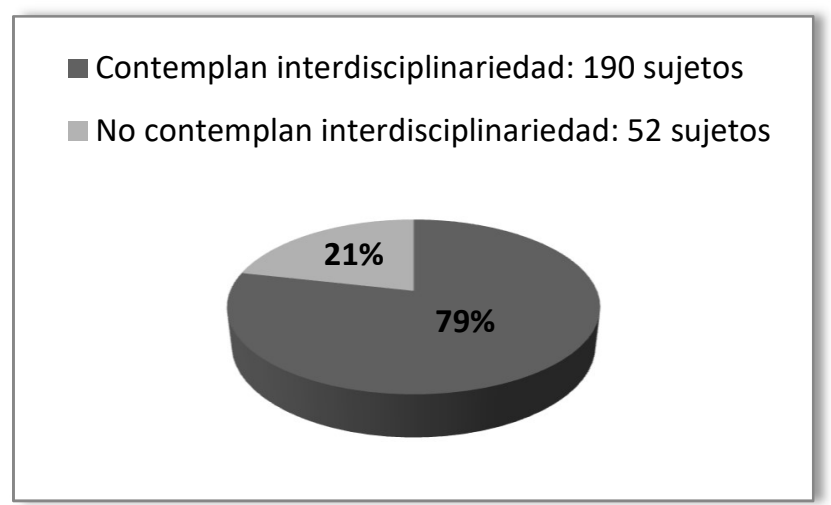

Figura 3. Número de participantes que contemplan estrategias favorecedoras de la interdisciplinariedad en sus programaciones de aula

En lo que respecta a la formación continua, los encuestados son conscientes de la importancia de la misma, sin embargo, echan en falta cursos, seminarios, talleres... específicos sobre Educación Musical.

El número de participantes que utilizan libro de texto supera al de aquellos que no lo hacen, siendo en los cursos de $3^{\circ}$ a $6^{\circ}$ donde se dan los porcentajes más elevados $(69 \%$ de los participantes). Estos datos concuerdan con los ofrecidos por Fernández (2008), especialmente en los aspectos relacionados con el menor uso de libro de texto en los cursos $1^{\circ}$ y $2^{\circ}$ de Primaria y con los resultados proporcionados por Vicente (2010), en cuanto a la gran aceptación del libro de texto por parte del profesorado especialista de música en esta etapa educativa.

NARCISO JOSÉ LÓPEZ GARCÍA. THE CONTENT OF THIS ARTICLE IS THE SOLE RESPONSIBILITY OF THE AUTHORS. THE REVISTA ELECTRÓNICA DE LEEME AND UNIVERSITAT DE VALĖNCIA ARE NOT LIABLE FOR ANY LEGAL ACTIONS THAT MAY ARISE INVOLVING THE ARTICLE'S CONTENT. REVISTA ELECTRÓNICA DE LEEME - LISTA ELECTRÓNICA EUROPEA DE MÚSICA EN LA EDUCACIÓN-HTTP://MUSICA.REDIRIS.ES.ISSN: 1575-9563 EDITORES: UNIVERSIDAD DE VALENCIA Y JESÚS TEJADA GIMÉNEZ. VISIBILIDAD DE ESTA REVISTA: EBSCO, CINDOC (CESIC), CITEFACTOR, COPAC, DIALNET, DICE (CSIC), DOAJ, E-REVISTAS (CSIC), EBSCO PREMIER, ERIH+, GALE CENGAGE LEARNING, IN-RECS, IRESIE, LATINDEX, MIAR, OCLC WORLDCAT, RESH, REDIB, RILM CORE JOURNALS, SUDOC, ULRICHS, ZEITSCHRIFTDATENBANK, ESTA REVISTA ESTÁ PUBLICADA CON EL APOYO INSTITUCIONAL DE REDIRISCCONSEJO SUPERIOR DE INVESTIGACIONES CIENTIFICAS Y ES DE ACCESO LIBRE. 
El 79\% de los docentes encuestados (190 sujetos) tienen aula específica, aunque las condiciones acústicas de éstas, son deficientes. Por otro lado, las aulas están bien equipadas, aunque el material es viejo y cuesta mucho renovarlo. Tienen ordenador, acceso a internet y, mayoritariamente, carecen de PDI.

Existe unanimidad a la hora de señalar que el material del que disponen es adecuado, excepto en la dotación digital, que es escasa y, en algunos casos, la que hay está obsoleta.

La percepción de los docentes sobre la opinión de sus claustros hacia la Educación Musical es que tanto los equipos directivos como el resto de compañeros apoyan la enseñanza de la música y se preocupan por el proceso de enseñanza-aprendizaje de sus alumnos en esta disciplina. Sin embargo, los equipos directivos no suelen ofrecer soporte económico para adquirir material nuevo para el aula.

En consonancia con los datos ofrecidos por López García (2010), los encuestados perciben aceptación y respeto de los claustros de sus centros hacia la asignatura de música, aunque estos desconocen los beneficios de la música en el desarrollo integral del niño en edad escolar. En esta misma línea, el 45\% opina que sus compañeros estarían de acuerdo en aumentar el tiempo lectivo de la Educación Musical si esto fuera posible. A su vez, una amplia mayoría, el $80 \%$ considera que las familias de sus centros no apoyan lo suficiente a esta asignatura como parte importante del currículo de Primaria y se preocupan muy poco por el proceso de enseñanza-aprendizaje de la música de sus hijos.

Se percibe que los alumnos disfrutan con la asignatura, pero esto no se ve reflejado en el número de alumnos que realizan actividades extraescolares relacionadas con la música.

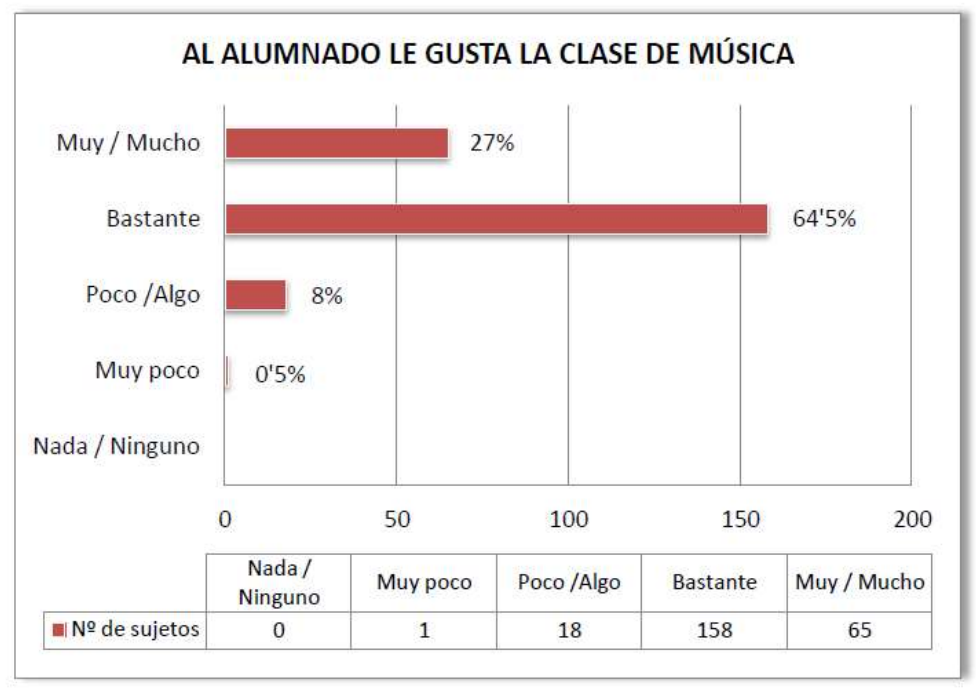

Figura 4. Percepción sobre la valoración del alumnado hacia la clase de música 


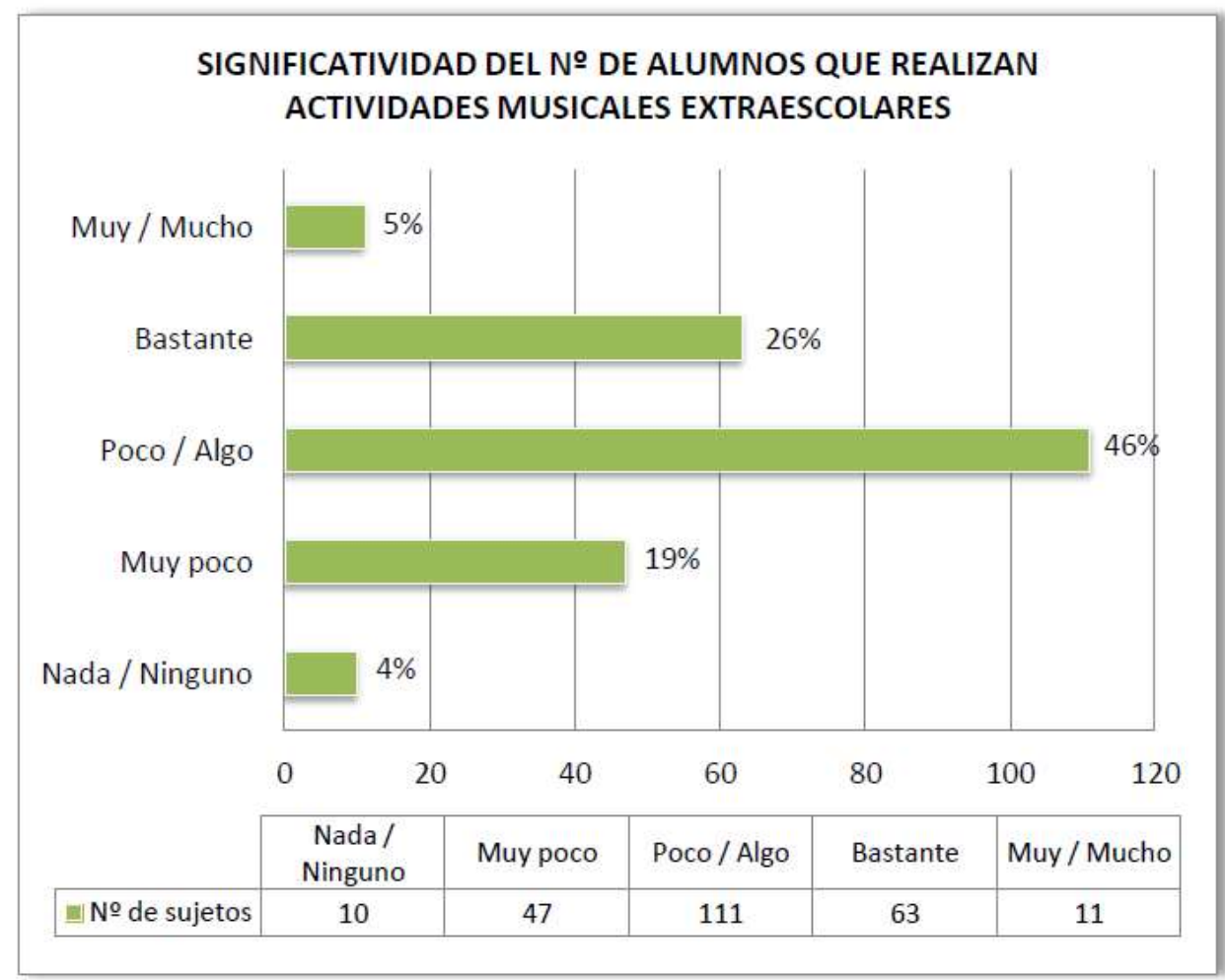

Figura 5. Significatividad del $\mathrm{n}^{\circ}$ de alumnos que realizan actividades musicales extraescolares

La percepción de los participantes sobre el nivel de valoración y respaldo de las administraciones hacia la enseñanza de la música es que éste es muy bajo. El 97\% piensa que el apoyo es poco o nulo.

Sobre si las administraciones educativas se preocupan por renovar y actualizar el material de música, el $98 \%$ señala que esta preocupación es prácticamente nula. Este colectivo echa de menos que las administraciones educativas les tenga en cuenta y que les pida opinión a la hora de modificar los currículos de esta materia. Es generalizada la opinión de que las últimas Leyes de Educación (LOGSE, LOCE, LOE y LOMCE) no han sabido gestionar adecuadamente la inclusión de la enseñanza de la música en la escuela.

El 54\% de los participantes, (131 sujetos) realiza actividades de formación o profesionales relacionadas con la música; aunque, en la mayoría de los casos, éstas no están vinculadas a la Educación Musical.

NARCISO JOSÉ LÓPEZ GARCÍA. THE CONTENT OF THIS ARTICLE IS THE SOLE RESPONSIBILITY OF THE AUTHORS. THE REVISTA ELECTRÓNICA DE LEEME AND UNIVERSITAT DE VALĖNCIA ARE NOT LIABLE FOR ANY LEGAL ACTIONS THAT MAY ARISE INVOLVING THE ARTICLE'S CONTENT. REVISTA ELECTRÓNICA DE LEEME -LISTA ELECTRÓNICA EUROPEA DE MÚSICA EN LA EDUCACIÓN-HTTP://MUSICA.REDIRIS. ES.ISSN: 1575-9563 EDITORES: UNIVERSIDAD DE VALENCIA Y JESÚS TEJADA GIMÉNEZ. VISIBILIDAD DE ESTA REVISTA: EBSCO, WORLDCAT, RESH, REDIB, RILM CORE DIALNET, DICE (CSIC), DOAJ, E-REVISTAS (CSIC), EBSCO PREMIER, ERIH+, GALE CENGAGE LEARNING, IN-RECS, IRESIE, LATINDEX, MIAR, OCLC SUPERIOR DE INVESTIGACIONES CIENTIFICAS Y ES DE ACCESO LIBRE. 


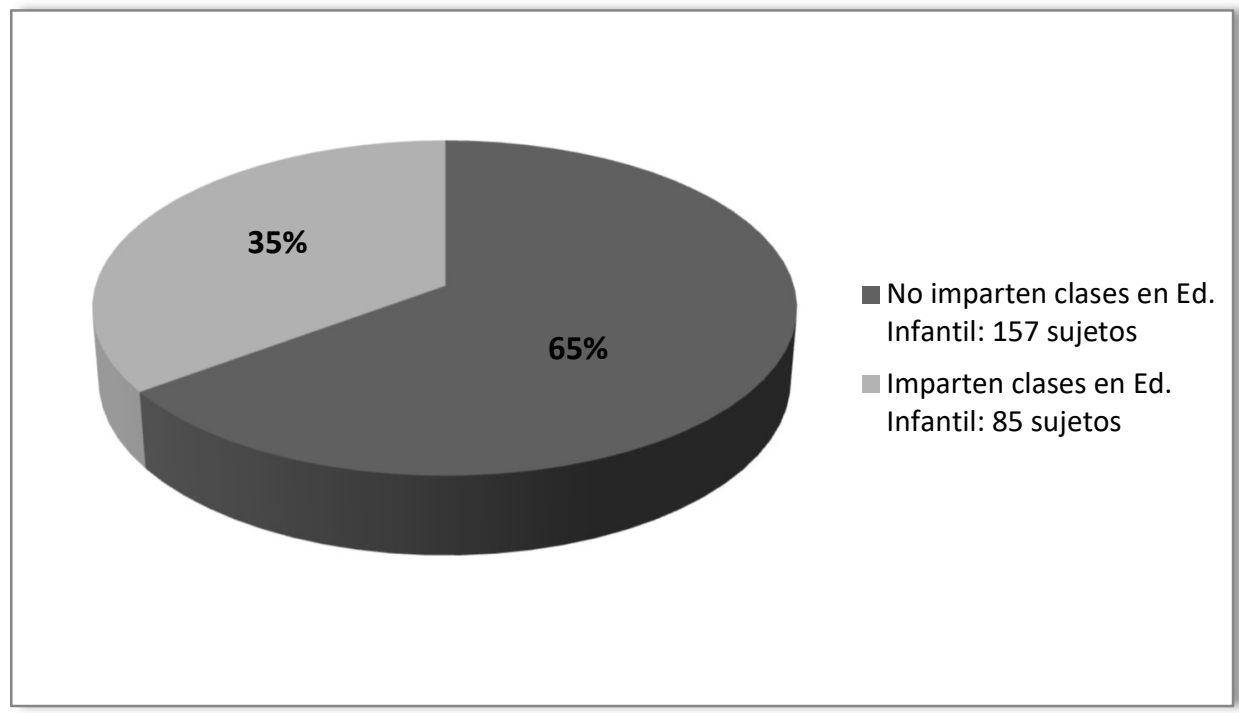

Figura 6. Número de docentes encuestados que imparten clases de música en Ed. Infantil

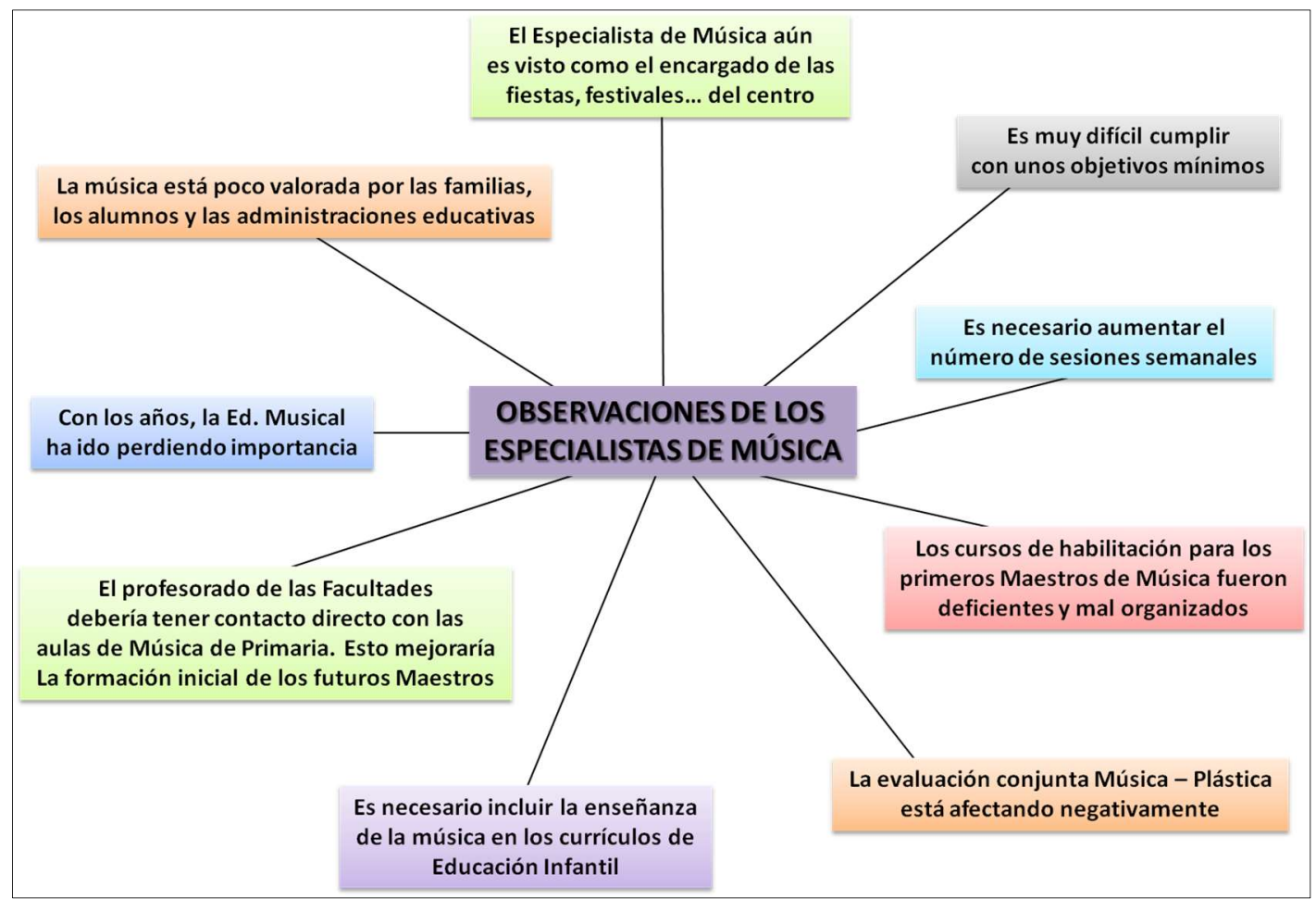

Figura 7. Observaciones recurrentes de los sujetos encuestados sobre la situación actual de la música en los centros de Primaria de Castilla - La Mancha

En relación a los niveles de dispersión obtenidos en las respuestas del tipo escala de Likert, hay que destacar que la desviación resultante ha sido muy baja (entre 0 y 0,9 ), por lo que las medias han aportado una descripción fiable de los datos extraídos. Sin embargo, se ha NARCISO JOSÉ LÓPEZ GARCÍA. THE CONTENT OF THIS ARTICLE IS THE SOLE RESPONSIBILITY OF THE AUTHORS. THE REVISTA ELECTRÓNICA DE LEEME AND UNIVERSITAT DE VALĖNCIA ARE NOT LIABLE FOR ANY LEGAL ACTIONS THAT MAY ARISE INVOLVING THE ARTICLE'S CONTENT. REVISTA ELECTRONIICA DE LEFME - LISTA ELECTRÓNICA EUROPEA DE MÚSICA EN LA EDUCACIÓN.HTTP://MUSICA.REDIRIS.ES.ISSN: 1575-9563 EDITORES: UNIVERSIDAD DE VALENCIA Y JESÚS TEJADA GIMÉNEZ. VISIBILIDAD DE ESTA REVISTA: EBSCO, CINDOC (CESIC), CITEFACTOR, COPAC, DIALNET, DICE (CSIC), DOAJ, E-REVISTAS (CSIC), EBSCO PREMIER, ERIH+, GALE CENGAGE LEARNING, IN-RECS, IRESIE, LATINDEX, MIAR, OCLC WORLDCAT, RESH, REDIB, RILM CORE JOURNALS, SUDOC, ULRICHS, ZEITSCHRIFTDATENBANK, ESTA REVISTA ESTÁ PUBLICADA CON EL APOYO INSTITUCIONAL DE REDIRIS-CONSEJO SUPERIOR DE INVESTIGACIONES CIENTIFICAS Y ES DE ACCESO LIBRE. 
comprobado que las medias más elevadas (entre 1,0 y 1,6 ) coinciden con las cuestiones relacionadas con el grado de adecuación de los recursos materiales (mobiliario, estado del aula, dotación digital e informática) y con el uso de las TIC en los procesos de enseñanza-aprendizaje de esta materia. Con respecto al primer supuesto, esta situación es debida a las importantes diferencias existentes entre los centros del ámbito rural, especialmente los ubicados en poblaciones con menos de 10.000 habitantes, y aquellos que se encuentran en las capitales de las cinco provincias y en las localidades mayores de 10.000 habitantes. Por otro lado, el nivel de dispersión relacionado con el uso de las TIC en el aula se debe al alto porcentaje de mujeres que se coloca en las franjas intermedias, que supera en más de 10 puntos al número de hombres.

Tabla 4. Desviación típica y media para la variable uso de las TIC en los procesos de enseñanza-aprendizaje de la música

\begin{tabular}{|c|c|c|c|}
\hline & $\mathrm{N}$ & Media & Desv. típ. \\
\hline uso_tic & 242 & 3,54 & 1,109 \\
N válido (según lista) & 242 & & \\
\hline
\end{tabular}

Tabla 5. Porcentajes y correlación entre las variables uso de las TIC en los procesos de enseñanza-aprendizaje de la música/sexo de los participantes

\begin{tabular}{|c|c|c|c|c|c|c|c|c|}
\hline & \multicolumn{5}{|c|}{ uso_tic } & \multirow[b]{2}{*}{ Total } \\
\hline & & & nada & muy poco & poco/algo & bastante & mucho & \\
\hline \multirow[t]{8}{*}{ sexo } & hombre & Recuento & 5 & 13 & 24 & 35 & 29 & 106 \\
\hline & & $\%$ dentro de sexo & $4,7 \%$ & $12,3 \%$ & $22,6 \%$ & $33,0 \%$ & $27,4 \%$ & $100,0 \%$ \\
\hline & & $\%$ dentro de uso_tic & $38,5 \%$ & $43,3 \%$ & $38,7 \%$ & $39,8 \%$ & $59,2 \%$ & $43,8 \%$ \\
\hline & & $\%$ del total & $2,1 \%$ & $5,4 \%$ & $9,9 \%$ & $14,5 \%$ & $12,0 \%$ & $43,8 \%$ \\
\hline & mujer & Recuento & 8 & 17 & 38 & 53 & 20 & 136 \\
\hline & & $\%$ dentro de sexo & $5,9 \%$ & $12,5 \%$ & $27,9 \%$ & $39,0 \%$ & $14,7 \%$ & $100,0 \%$ \\
\hline & & $\%$ dentro de uso_tic & $61,5 \%$ & $56,7 \%$ & $61,3 \%$ & $60,2 \%$ & $40,8 \%$ & $56,2 \%$ \\
\hline & & $\%$ del total & $3,3 \%$ & $7,0 \%$ & $15,7 \%$ & $21,9 \%$ & $8,3 \%$ & $56,2 \%$ \\
\hline \multirow{4}{*}{\multicolumn{2}{|c|}{ Total }} & Recuento & 13 & 30 & 62 & 88 & 49 & 242 \\
\hline & & $\%$ dentro de sexo & $5,4 \%$ & $12,4 \%$ & $25,6 \%$ & $36,4 \%$ & $20,2 \%$ & $100,0 \%$ \\
\hline & & $\%$ dentro de uso_tic & $100,0 \%$ & $100,0 \%$ & $100,0 \%$ & $100,0 \%$ & $100,0 \%$ & $100,0 \%$ \\
\hline & & $\%$ del total & $5,4 \%$ & $12,4 \%$ & $25,6 \%$ & $36,4 \%$ & $20,2 \%$ & $100,0 \%$ \\
\hline
\end{tabular}

NARCISO JOSÉ LÓPEZ GARCÍA. THE CONTENT OF THIS ARTICLE IS THE SOLE RESPONSIBILITY OF THE AUTHORS. THE REVISTA ELECTRÓNICA DE LEEME AND UNIVERSITAT DE VALĖNCIA ARE NOT LIABLE FOR ANY LEGAL ACTIONS THAT MAY ARISE INVOLVING THE ARTICLE'S CONTENT. REVISTA ELECTRÓNICA DE LEEME -LISTA ELECTRÓNICA EUROPEA DE MÚSICA EN LA EDUCACIÓN-HTTP://MUSICA.REDIRIS.ES.ISSN: 1575-9563 EDITORES: UNIVERSIDAD DE VALENCIA Y JESÚS TEJADA GIMÉNEZ. VISIBILIDAD DE ESTA REVISTA: EBSCO, CINDOC (CESIC), CITEFACTOR, COPAC, DIALNET, DICE (CSIC), DOAJ, E-REVISTAS (CSIC), EBSCO PREMIER, ERIH+, GALE CENGAGE LEARNING, IN-RECS, IRESIE, LATINDEX, MIAR, OCLC WORLDCAT, RESH, REDIB, RILM CORE JOURNALS, SUDOC, ULRICHS, ZEITSCHRIFTDATENBANK, ESTA REVISTA ESTÁ PUBLICADA CON EL APOYO INSTITUCIONAL DE REDIRIS-CONSEJO SUPERIOR DE INVESTIGACIONES CIENTIFICAS Y ES DE ACCESO LIBRE. 


\subsection{La Técnica Delphi: datos relevantes}

Una vez realizada la Técnica Delphi y, tras recoger, analizar y ordenar jerárquicamente las respuestas de los participantes, se extrajeron las conclusiones pertinentes, clasificadas en los cuatro bloques temáticos en los que se dividió esta técnica.

Tabla 6. Conclusiones derivadas de la Técnica Delphi

\section{Sobre el estado actual de la Educación Musical en Primaria}

La evaluación compartida Plástica - Música está perjudicando el desarrollo normalizado de esta materia

Una sesión semanal complica llevar a cabo de manera efectiva los programas y, por tanto, conseguir los objetivos mínimos establecidos

Se percibe cierto abandono por parte de las administraciones educativas hacia la enseñanza de la música en esta etapa educativa

Las condiciones actuales dificultan enormemente una mejor enseñanza musical

En la mayoría de los centros no existen los recursos espaciales y materiales adecuados a los nuevos tiempos educativos

El analfabetismo musical de la sociedad española repercute negativamente en el pleno desarrollo de esta disciplina en las escuelas

Las familias no contemplan la ed. Musical desde una posición de igualdad con respecto al resto de materias del currículo

En demasiadas ocasiones la comunidad educativa espera del especialista de música que se haga cargo de preparar diferentes actividades en forma de festivales, conciertos, fiestas escolares, etc.

\section{Sobre los cambios que se deberían llevar a cabo para la mejora de la enseñanza de la música en Primaria}

La evaluación de Plástica y Música debería ser independiente, teniendo cada materia un apartado independiente tanto en el boletín informativo como en el programa informático de gestión de los centros

Se deberían tener más en cuenta las opiniones y experiencias del profesorado

La pizarra digital y otras herramientas TIC deberían estar presentes en todas las aulas de música

Aumento de sesiones semanales, al menos en $5^{\circ}$ y $6^{\circ}$ cursos

Compromiso de las administraciones educativas por renovar el material deteriorado y por adaptarlo a los nuevos cambios educativos

Inclusión de la Educación Musical en la etapa de Educación Infantil

Los estudios de Magisterio deberían ofrecer una formación más completa

Creación de aulas acondicionadas y dotadas para impartir la especialidad, sin que estén en peligro según las necesidades de los centros

El profesorado de Magisterio debería estar más relacionado con la escuela Primaria, no tanto con la Secundaria y con los Conservatorios

NARCISO JOSÉ LÓPEZ GARCÍA. THE CONTENT OF THIS ARTICLE IS THE SOLE RESPONSIBILITY OF THE AUTHORS. THE REVISTA ELECTRÓNICA DE LEEME AND UNIVERSITAT DE VALĖNCIA ARE NOT LABLE FOR ANY LEGA ACTIONS THAT MAY ARISE INVOLVING THE ARTICLE'S CONTENT. REVISTA ELECTRÓNCA DE LEEME -LISTA ELECTRÓNICA EUROPEA DE MÚSICA EN LA EDUCACIÓN-HTTP://MUSICA.REDIRIS.ES.ISSN: 1575-9563 EDITORES: UNIVERSIDAD DE VALENCIA Y JESÚS TEJADA GIMÉNEZ. VISIBILIDAD DE ESTA REVISTA: EBSCOO, CINDOC (CESIC), CITEFACTOR, COPAC, DIALNET, DICE (CSIC), DOAJ, E-REVISTAS (CSIC), EBSCO PREMIER, ERIH+, GALE CENGAGE LEARNING, IN-RECS, IRESIE, LATINDEX, MIAR, OCLC WORLDCAT, RESH, REDIB, RILM CORE JOURNALS, SUDOC, ULRICHS, ZEITSCHRIFTDATENBANK, ESTA REVISTA ESTÁ PUBLICADA CON EL APOYO INSTITUCIONAL DE REDIRIS-CONSEJO SUPERIOR DE INVESTIGACIONES CIENTIFICAS Y ES DE ACCESO LIBRE. 
LISTA ELECTRÓNICA EUROPEA DE MÚSICA EN LA EDUCACIÓN

Sería fundamental la comunicación fluida con los IES para dar continuidad a los aprendizajes del alumnado Sobre el peso de la Educación Musical en la legislación vigente

Existe un abismo entre materias instrumentales y materias complementarias

Las Leyes posteriores a la LOGSE no han sabido gestionar adecuadamente la evolución y desarrollo de esta materia en los currículos

Las reformas legislativas deberían recoger la opinión de los profesionales en la materia: los docentes

Sobre la opinión de la comunidad educativa acerca de la Educación Musical

El analfabetismo musical de las familias perjudica enormemente a la música en las escuelas

En casa, la música no está suficientemente valorada, y los padres, en general, no están capacitados para ayudar a sus hijos

En demasiados casos, existe una despreocupación de los padres por que sus hijos lleven el material de música a clase, repasen los conocimientos que van adquiriendo o suspendan la materia

Son frecuentes los padres que justifican el poco interés que muestran sus hijos ante esta disciplina

En general, la comunidad educativa piensa que es una asignatura de relleno, sin valor pedagógico ni formativo.

Con respecto a la percepción sobre la opinión del alumnado el resultado es más favorable y, generalmente, se aprecia bastante motivación e interés

\subsection{El cuestionario CE-02: datos relevantes}

Diez de los profesores de las Facultades de Educación de la UCLM que han realizado el cuestionario señalan estar de acuerdo con el hecho de que el profesorado especialista de música de Primaria complete su horario ejerciendo labores de tutor.

Este profesorado percibe que el número y calidad de instrumentos musicales escolares de las aulas de música de los centros de Primaria de nuestra región no se adapta a las necesidades pedagógico-musicales actuales.

Los encuestados perciben que la opinión de la comunidad educativa sobre la Educación Musical en Primaria es buena o muy buena. Sólo tres participantes señalaron que percibían una situación negativa hacia la enseñanza de la música en las escuelas.

La mayoría de participantes, 11 personas, manifestó que la LOGSE impulsó adecuadamente la inserción de la Educación Musical en esta etapa educativa. Sin embargo, la opinión de los participantes varía significativamente con respecto a la LOE; 9 encuestados están en desacuerdo con los aspectos establecidos por esta Ley para el correcto desarrollo de los procesos de enseñanza-aprendizaje de la música en Primaria.

Por otro lado, conociendo los fuertes cambios estructurales realizados por la actual Ley de Educación (LOMCE), el 67\% del profesorado encuestado (10 participantes) opina que las

NARCISO JOSÉ LÓPEZ GARCÍA. THE CONTENT OF THIS ARTICLE IS THE SOLE RESPONSIBILITY OF THE AUTHORS. THE REVISTA ELECTRÓNICA DE LEEME AND UNIVERSITAT DE VALĖNCIA ARE NOT LIABLE FOR ANY LEGAL ACTIONS THAT MAY ARISE INVOLVING THE ARTICLE'S CONTENT. REVISTA ELECTRÓNICA DE LEEME - LISTA ELECTRÓNICA EUROPEA DE MÚSICA EN LA EDUCACIÓN-HTTP://MUSICA.REDIRIS.ES.ISSN: 1575-9563 EDITORES: UNIVERSIDAD DE VALENCIA Y JESÚS TEJADA GIMÉNEZ. VISIBILIDAD DE ESTA REVISTA: EBSCO, CINDOC (CESIC), CITEFACTOR, COPAC, DIALNET, DICE (CSIC), DOAJ, E-REVISTAS (CSIC), EBSCO PREMIER, ERIH+, GALE CENGAGE LEARNING, IN-RECS, IRESIE, LATINDEX, MIAR, OCLC WORLDCAT, RESH, REDIB, RILM CORE JOURNALS, SUDOC, ULRICHS, ZEITSCHRIFTDATENBANK, ESTA REVISTA ESTÁ PUBLICADA CON EL APOYO INSTITUCIONAL DE REDIRISCCONSEJO SUPERIOR DE INVESTIGACIONES CIENTIFICAS Y ES DE ACCESO LIBRE. 
modificaciones curriculares llevadas a cabo en esta materia no supondrán un retroceso en la evolución de la misma en esta etapa educativa.

Con respecto a la conveniencia de establecer la enseñanza obligatoria de la música en la etapa de Educación Infantil, el 75\% (11 sujetos) estaría de acuerdo con esta premisa.

Por último, 11 encuestados opinan que las plazas itinerantes-compartidas pueden afectar negativamente en el correcto desarrollo de los procesos de enseñanza-aprendizaje de la música en la escuela, influyendo desfavorablemente en la labor docente de los maestros especialistas.

Tabla 7. Observaciones generales realizadas por el profesorado de la UCLM Observaciones del profesorado de la UCLM

Horario insuficiente, escaso material, poca cooperación con otras áreas y escasa formación continua del profesorado

La Educación Musical en Primaria nunca ha sido lo suficientemente adecuada a pesar de los esfuerzos que se han realizado

Una hora a la semana es absolutamente insuficiente para lograr determinados objetivos en el desarrollo de la percepción y la expresión musical

Normalmente, el especialista da clases a todos los alumnos del colegio, por lo que ve cada semana a muchos grupos sobre los que no llega a tener el conocimiento y el control necesarios

La administración ha mirado con malos ojos a la música en demasiadas ocasiones

Muchos docentes no son conscientes de la importancia de este hecho cultural en el desarrollo de los niños y no apoyan o ayudan desde otros ángulos al profesor de música

El área de Educación Artística debería ser impartida por un maestro competente en Plástica y Música. Ello permitiría al profesorado un mejor conocimiento de los diferentes grupos de alumnos y, a su vez, propiciaría abordar las enseñanzas artísticas de una forma globalizada mediante proyectos integrados de Plástica y Música.

\section{Conclusiones}

Tras el análisis e interpretación de los datos obtenidos, se exponen las conclusiones más significativas derivadas del proceso de investigación. Éstas recogen, en líneas generales, los aspectos más relevantes relacionados con la realidad tanto del profesorado de Educación Musical en su labor docente (particularidades, necesidades y demandas más representativas) como de la materia que imparten. Además, se exponen las inferencias más significativas derivadas del proceso de triangulación.

Este profesorado es joven, con menos de 15 años de experiencia docente en la mayoría de los casos; aunque, a priori, muy cualificado, ya que posee un importante bagaje en formación musical y la titulación específica de maestro de música. Sin embargo, coincidiendo con estudios similares (González y Fuentes, 2011; Rosa ,2015; y Vilar y Gómez, 2005, entre otros) opina que

NARCISO JOSÉ LÓPEZ GARCÍA. THE CONTENT OF THIS ARTICLE IS THE SOLE RESPONSIBLITY OF THE AUTHORS. THE REVISTA ELECTRÓNICA DE LEEME AND UNIVERSITAT DE VALĖNCIA ARE NOT LIABLE FOR ANY LEGAL ACTIONS THAT MAY ARISE INVOLVING THE ARTICLE'S CONTENT. REVISTA ELECTRÓNICA DE LEEME - LISTA ELECTRÓNICA EUROPEA DE MÚSICA EN LA EDUCACIÓN-HTTP://MUSICA.REDIRIS.ES.ISSN: 1575-9563 EDITORES: UNIVERSIDAD DE VALENCIA Y JESÚS TEJADA GIMÉNEZ. VISIBILIDAD DE ESTA REVISTA: EBSCOO, CINDOC (CESIC), CITEFACTOR, COPAC, DIALNET, DICE (CSIC), DOAJ, E-REVISTAS (CSIC), EBSCO PREMIER, ERIH+, GALE CENGAGE LEARNING, IN-RECS, IRESIE, LATINDEX, MIAR, OCLC WORLDCAT, RESH, REDIB, RILM CORE JOURNALS, SUDOC, ULRICHS, ZEITSCHRIFTDATENBANK, ESTA REVISTA ESTÁ PUBLICADA CON EL APOYO INSTITUCIONAL DE REDIRISCCONSEJO SUPERIOR DE INVESTIGACIONES CIENTIFICAS Y ES DE ACCESO LIBRE. 
la formación inicial estuvo más centrada en la adquisición de conocimientos académicos que de aptitudes didácticas y musicales relacionadas con la realidad del aula, provocando ciertos desajustes que en su opinión deberían ser analizados y subsanados.

Existe una gran estabilidad en las plantillas, lo que favorece tanto el desarrollo educativo-musical de los alumnos como el propio desarrollo de la materia.

Mientras que en los centros privados y concertados ejercen mayoritariamente labores de tutor, en los centros públicos no es habitual este tipo de funciones, ya sea porque se completa el horario con sesiones de apoyo o impartiendo algunas otras asignaturas distintas a la de música, o bien porque su plaza es compartida-itinerante. Este aspecto, que se desarrolla con más detenimiento en el segundo bloque de conclusiones, y sus posibles repercusiones en la práctica docente, es uno de los puntos contrastantes más llamativos surgidos del proceso de triangulación de los datos obtenidos.

Los participantes reconocen no tener un conocimiento profundo de la estructura curricular actual basada en la consecución de competencias. Esta carencia abre las puertas a investigaciones futuras que puedan aclarar, entre otros asuntos, si existe una desconexión entre la práctica docente y la legislación educativa que pueda estar afectando negativamente a los procesos de enseñanza-aprendizaje. Además, los sujetos que han participado en nuestro estudio echan en falta una formación sólida sobre cómo programar y cómo evaluar atendiendo a esta nueva configuración del currículo, como pone también de manifiesto el trabajo realizado por Vázquez-Cano (2016), coincidiendo en afirmar que las tareas burocráticas se han convertido en un verdadero problema, entorpeciendo la labor de aula y restando tiempo al profesorado en las pocas sesiones que disponen para preparar sus clases.

En lo referente a infraestructuras y recursos materiales, las condiciones se adecuan y son suficientes para afrontar el marco curricular diseñado para esta materia. Sin embargo, se percibe cierto abandono por parte de las administraciones educativas a la hora de renovar el material del que se dispone, especialmente instrumentos musicales, así como en la compra de nuevos equipos adaptados a las necesidades sociales y educativas actuales. Como consecuencia más notable de lo anterior, un buen número de participantes reconoce que sus aulas se van quedando obsoletas, hasta el punto de preferir impartir sus clases en las aulas de Primaria, lo que les permite aprovechar los equipamientos TIC de estos espacios.

La incorporación de la Educación Musical en los currículos oficiales se llevó a cabo, desde un primer momento, en condiciones muy diferentes al resto de materias consideradas fundamentales. Este hecho, que no se ha subsanado con el paso de los años, ha favorecido la escasa consideración tanto de las familias como de las administraciones educativas hacia esta disciplina. Sin duda, es la percepción de este colectivo sobre la opinión de las administraciones educativas y su interés por mejorar las condiciones curriculares de la enseñanza de la música en esta etapa educativa la que genera más críticas negativas. Este colectivo docente considera que

NARCISO JOSÉ LÓPEZ GARCÍA. THE CONTENT OF THIS ARTICLE IS THE SOLE RESPONSIBILITY OF THE AUTHORS. THE REVISTA ELECTRÓNICA DE LEEME AND UNIVERSITAT DE VALĖNCIA ARE NOT LIABLE FOR ANY LEGAL ACTIONS THAT MAY ARISE INVOLVING THE ARTICLE'S CONTENT. REVISTA ELECTRÓNICA DE LEEME - LISTA ELECTRÓNICA EUROPEA DE MÚSICA EN LA EDUCACIÓN-HTTP://MUSICA.REDIRIS.ES.ISSN: 1575-9563 EDITORES: UNIVERSIDAD DE VALENCIA Y JESÚS TEJADA GIMÉNEZ. VISIBILIDAD DE ESTA REVISTA: EBSCO, CINDOC (CESIC), CITEFACTOR, COPAC, DIALNET, DICE (CSIC), DOAJ, E-REVISTAS (CSIC), EBSCO PREMIER, ERIH+, GALE CENGAGE LEARNING, IN-RECS, IRESIE, LATINDEX, MIAR, OCLC WORLDCAT, RESH, REDIB, RILM CORE JOURNALS, SUDOC, ULRICHS, ZEITSCHRIFTDATENBANK, ESTA REVISTA ESTÁ PUBLICADA CON EL APOYO INSTITUCIONAL DE REDIRISCCONSEJO SUPERIOR DE INVESTIGACIONES CIENTIFICAS Y ES DE ACCESO LIBRE. 
el grado de implicación de las instituciones competentes es inexistente, lo que está perjudicando gravemente los procesos de enseñanza-aprendizaje de la música, así como su labor docente.

Coincidiendo con los trabajos de Álvarez (2004), Comellas (2009), Martí (2008), Pastor (2008) y Schafer (2008), entre otros, en este momento podemos afirmar que las relaciones entre el profesorado especialista, las familias y las administraciones son poco estimulantes y poco colaborativas, lo que puede convertirse en una barrera que impida o frene aún más el desarrollo normal y efectivo de la enseñanza de la música en la escuela. Es prioritario por tanto replantearse el papel de cada uno de los agentes que componen la comunidad educativa ya que, como señala Gil (1994), la participación del conjunto de componentes de la comunidad educativa es clave y se convierte en una de las pocas vías que posibilitan la construcción de un tejido social capaz de solventar algunos de los problemas de aprendizaje que se suelen dar en los primeros años de escolarización.

El profesorado de los centros privados o concertados percibe una mayor aceptación de la comunidad educativa hacia la Educación Musical. Los resultados obtenidos señalan que estos docentes tienen una opinión más positiva sobre la valoración de las familias hacia la enseñanza de la música. Además, éstas parecen mostrar mayor interés hacia el proceso de enseñanzaaprendizaje de sus hijos en esta asignatura.

Uno de los problemas más recurrentes está relacionado con la exigua dedicación horaria que se ha otorgado a esta materia, desde su incorporación obligatoria a los currículos oficiales hasta el momento actual. Por otro lado, los especialistas vienen reclamando una solución al proceso de evaluación conjunta al que se ve sometida esta disciplina, lo que ha contribuido a mantener la visión sesgada que las familias y las administraciones tienen sobre la misma. En estas dos cuestiones, los reducidos horarios y la evaluación conjunta, el profesorado encuestado vuelve a coincidir con los resultados obtenidos en un buen número de investigaciones como son los trabajos de De Benito (2012), Domínguez (2014), Montesinos (2009/2010), Ocaña (2006) y Reyes (2015), entre otros.

Desde nuestro punto de vista y en base a los datos obtenidos de las observaciones proporcionadas por los participantes en el CE-01 (Figura 7) y de la Técnica Delphi (Tabla 4), esta falta de peso específico de la música a nivel evaluativo puede ser una causa de desmotivación del profesorado y de despreocupación ante el correcto desarrollo de los procesos de aprendizaje de los alumnos, tanto de las familias como de las instituciones educativas.

Con respecto a la formación permanente, si bien una amplia mayoría de participantes tiene formación musical y, actualmente, está realizando algún tipo de actividad relacionada con la música, son muchos los que echan en falta una oferta mayor y más atractiva sobre formación en didáctica de la Educación Musical.

En relación al tratamiento que ha tenido la Educación Musical en las normas educativas, este colectivo piensa que, hasta la fecha, ninguna ley de Educación posterior a la LOGSE ha garantizado el desarrollo efectivo de la enseñanza de la música a lo largo de los años, haciendo NARCISO JOSÉ LÓPEZ GARCÍA. THE CONTENT OF THIS ARTICLE IS THE SOLE RESPONSIBILITY OF THE AUTHORS. THE REVISTA ELECTRÓNICA DE LEEME AND UNIVERSITAT DE VALĖNCIA ARE NOT LIABLE FOR ANY LEGAL ACTIONS THAT MAY ARISE INVOLVING THE ARTICLE'S CONTENT. REVISTA ELECTRÓNICA DE LEEME -LISTA ELECTRÓNICA EUROPEA DE MÚSICA EN LA EDUCACIÓN-HTTP://MUSICA.REDIRIS. ES.ISSN: 1575-9563 EDITORES: UNIVERSIDAD DE VALENCIA Y JESÚS TEJADA GIMÉNEZ. VISIBILIDAD DE ESTA REVISTA: EBSCO, WORLDCAT, RESH, REDIB, RILM CORE JOURNALS, SUDOC, ULRICHS, ZEITSCHRIFTDATENBANK, ESTA REVISTA ESTÁ PUBLICADA CON EL APOYO INSTITUCIONAL DE REDIRIS-CONSEJO SUPERIOR DE INVESTIGACIONES CIENTIFICAS Y ES DE ACCESO LIBRE. 
renacer una y otra vez el temor de que se reduzca su espacio por razones políticas y/o económicas. Una vez más, la opinión de los participantes converge con los datos aportados por otros expertos como De Benito (2012), Pastor (2004), Pliego (2008) y Roche (2010), en relación a la LOGSE, Ocaña (2006) y Reyes (2005), en relación a la LOCE y a la LOE y Domínguez (2014), que aporta interesantes datos sobre la situación actual de la Educación Musical en el contexto educativo de la LOMCE.

La percepción generalizada de los maestros de música de las escuelas castellanomanchegas es que los ámbitos institucional y legislativo no han sabido ofrecer, hasta la fecha, mecanismos que respondan a las demandas y necesidades planteadas desde el ámbito experiencial de la realidad del aula. Además, las administraciones educativas han desarrollo constantes cambios en la normativa sin tener en cuenta la opinión de este colectivo, lo que está provocando desmotivación e indiferencia de este profesorado ante las diferentes reformas educativas que se vienen sucediendo con demasiada frecuencia.

Por otro lado, la triangulación metodológica utilizada en esta investigación ha proporcionado dos interpretaciones diferentes de los resultados obtenidos. Por un lado, el CE-01 y la Técnica Delphi han arrojado información redundante cumpliéndose así una de las principales finalidades de los procesos de triangulación, consistente en esclarecer y verificar aspectos significativos del estudio (Okuda y Gómez-Restrepo, 2005). Por otro lado, los cuestionarios CE-01 y CE-02 han ofrecido una serie de datos contrastantes especialmente interesantes. Este hecho, en contra de lo que podría parecer y teniendo en cuenta, de nuevo, las palabras de Okuda y Gómez-Restrepo (2005), han enriquecido sobremanera este estudio y han brindado la oportunidad de vislumbrar vías de investigación futuras.

Teniendo en cuenta que el CE-01 lo han cumplimentado los maestro de música de los centros de Primaria de Castilla-La Mancha y el CE-02 el Profesorado de los Departamentos de Expresión Musical de las cuatro Facultades de Educación de la UCLM, se ha observado que en algunos temas se da una perspectiva diferente entre la realidad del aula y los procesos de formación inicial y en la percepción de cada uno de estos colectivos con respecto a cuatro temas fundamentales:

1. La realización de labores de tutor por parte del especialista de música: mientras que los maestros están en desacuerdo y demandan más sesiones de Educación Musical en cada grupo-curso y más tiempo para la preparación de sus clases, el profesorado de la UCLM está bastante de acuerdo en que los maestros ejerzan este tipo de funciones, lo que da pie a pensar que el profesorado de las Facultades de Educación ve más apropiado que un maestro de música ejerza ambas labores en el mismo centro a que tenga que ocupar una plaza compartida-itinerante, lo que desde su punto de vista perjudica los procesos de enseñanza-aprendizaje de la música.

2. Las dotaciones de las aulas, especialmente las de instrumentos Orff: los especialistas valoran positivamente tanto el grado de adecuación de las mismas como su estado actual. Sin embargo, son conscientes de que el contexto educativo en estos momentos

NARCISO JOSÉ LÓPEZ GARCÍA. THE CONTENT OF THIS ARTICLE IS THE SOLE RESPONSIBILITY OF THE AUTHORS. THE REVISTA ELECTRÓNICA DE LEEME AND UNIVERSITAT DE VALĖNCIA ARE NOT LIABLE FOR ANY LEGAL ACTIONS THAT MAY ARISE INVOLVING THE ARTICLE'S CONTENT. REVISTA ELECTRÓNICA DE LEEME -LISTA ELECTRÓNICA EUROPEA DE MÚSICA EN LA EDUCACIÓN-HTTP://MUSICA.REDIRIS.ES.ISSN: 1575-9563 EDITORES: UNIVERSIDAD DE VALENCIA Y JESÚS TEJADA GIMÉNEZ. VISIBILIDAD DE ESTA REVISTA: EBSCO, CINDOC (CESIC), CITEFACTOR, COPAC, DIALNET, DICE (CSIC), DOAJ, E-REVISTAS (CSIC), EBSCO PREMIER, ERIH+, GALE CENGAGE LEARNING, IN-RECS, IRESIE, LATINDEX, MIAR, OCLC WORLDCAT, RESH, REDIB, RILM CORE JOURNALS, SUDOC, ULRICHS, ZEITSCHRIFTDATENBANK, ESTA REVISTA ESTÁ PUBLICADA CON EL APOYO INSTITUCIONAL DE REDIRISCCONSEJO SUPERIOR DE INVESTIGACIONES CIENTIFICAS Y ES DE ACCESO LIBRE. 
está demandando la inclusión de otro tipo de materiales. Por su parte, el profesorado de la UCLM no es tan positivo en su valoración y echa en falta materiales acordes a las tendencias más actuales de este campo de conocimiento.

3. El impulso de la LOGSE a la Educación Musical: El colectivo de docentes de la UCLM tiene una valoración muy positiva con respecto a este tema. Contrariamente, la postura de los especialistas no es tan favorable, existiendo un alto porcentaje de docentes de Primaria que opina que el impulso a la enseñanza de la música no fue todo lo positivo que se esperaba.

4. La opinión de la comunidad educativa sobre la Educación Musical en Primaria: la desfavorable percepción de los especialistas sobre la valoración de su asignatura por parte de la comunidad educativa (especialmente de las familias y administraciones educativas) no es compartida por el profesorado de la UCLM, que percibe que esta disciplina está bien considerada.

Esta situación, en la misma línea de los trabajos de González y Fuentes (2011), Rosa (2015) y Vilar y Gómez (2005), induce a pensar que falta un contacto directo más profundo entre la universidad y el aula de Primaria, y que este contacto proporcionaría a las facultades de Educación una visión más real y necesaria para planificar sus programas y planes de estudios.

Una vez conocidos, analizados y expuestos los resultados obtenidos, estamos en condiciones de afirmar que se han resuelto los interrogantes planteados para esta investigación y que se ha cumplido con el Objetivo General de la misma. Sin embargo, aún queda mucho camino por recorrer y que, en el contexto actual, la Educación Musical en Castilla-La Mancha y, por extensión, en el resto del territorio nacional, necesita someterse a un proceso de reflexión profunda con el que dar una respuesta firme y clarificadora tanto a nivel laboral - profesional como a nivel curricular. Para ello, es imprescindible un compromiso firme de las instituciones educativas de contar con las experiencias de los propios profesionales de la Educación Musical. Sólo de esta forma se podrán sentar las bases para configurar un currículo que tome en consideración la importancia de la música en el desarrollo de las capacidades y competencias clave de los niños en edad escolar.

La investigación educativa está sometida a una serie de parámetros que, por el dinamismo propio de los procesos de enseñanza-aprendizaje y por el advenimiento de cambios sociales, políticos, legislativos, pueden verse alterados de tal forma que se produzcan modificaciones en el contexto educativo a estudio una vez comenzado el proceso de investigación. En este caso, una de las limitaciones más importantes encontradas fue la publicación y puesta en marcha de una nueva Ley de Educación, la LOMCE, coincidiendo con las últimas fases del trabajo. Ésta ha modificado profundamente la estructura curricular de la enseñanza primaria $y$, especialmente, la configuración de la enseñanza de la música en esta etapa educativa, que ha dejado de ser una materia obligatoria. Esto obligó a modificar las herramientas de recogida de datos. Aun así, el CE-01 y la Técnica Delphi no recogen en profundidad la opinión de los especialistas de música de esta Comunidad Autónoma sobre este nuevo panorama educativo-musical.

NARCISO JOSÉ LÓPEZ GARCÍA. THE CONTENT OF THIS ARTICLE IS THE SOLE RESPONSIBILITY OF THE AUTHORS. THE REVISTA ELECTRÓNICA DE LEEME AND UNIVERSITAT DE VALĖNCIA ARE NOT LIABLE FOR ANY LEGAL ACTIONS THAT MAY ARISE INVOLVING THE ARTICLE'S CONTENT. REVISTA ELECTRÓNICA DE LEEME - LISTA ELECTRÓNICA EUROPEA DE MÚSICA EN LA EDUCACIÓN-HTTP://MUSICA.REDIRIS.ES.ISSN: 1575-9563 EDITORES: UNIVERSIDAD DE VALENCIA Y JESÚS TEJADA GIMÉNEZ. VISIBILIDAD DE ESTA REVISTA: EBSCO, CINDOC (CESIC), CITEFACTOR, COPAC, DIALNET, DICE (CSIC), DOAJ, E-REVISTAS (CSIC), EBSCO PREMIER, ERIH+, GALE CENGAGE LEARNING, IN-RECS, IRESIE, LATINDEX, MIAR, OCLC WORLDCAT, RESH, REDIB, RILM CORE JOURNALS, SUDOC, ULRICHS, ZEITSCHRIFTDATENBANK, ESTA REVISTA ESTÁ PUBLICADA CON EL APOYO INSTITUCIONAL DE REDIRISCCONSEJO SUPERIOR DE INVESTIGACIONES CIENTIFICAS Y ES DE ACCESO LIBRE. 
Otra limitación encontrada está relacionada con el acceso a la población a la que iba dirigido el CE-01. El hecho de contar con intermediarios -los equipos directivos de los centros públicos, privados y concertados- ha ralentizado demasiado el proceso de recogida de datos y ha dificultado el proceso de información, realización y devolución de los cuestionarios electrónicos destinados a los maestros de música, especialmente en los centros privados y concertados.

Además, la cantidad de información recogida, la naturaleza cualitativa de un buen número de respuestas y la realización de los análisis generales y correlacionales, ha forzado a dedicar un tiempo extra al programado para estas funciones. La falta de investigaciones similares llevadas a cabo en Castilla-La Mancha ha obligado a realizar una búsqueda de publicaciones análogas elaboradas en otras Comunidades Autónomas.

Por último, queda señalar la limitación de la muestra utilizada debido a la localización geográfica de la investigación. El hecho de centrarla en el colectivo de especialistas de música de los centros de Primaria de Castilla-La Mancha, si bien ha permitido dar respuesta a los interrogantes planteados y cumplir con los objetivos propuestos, es probable que adolezca de validez externa con la que poder realizar predicciones en el resto del colectivo del ámbito del territorio español, aunque hayan quedado comprobadas las semejanzas con trabajos análogos.

Con respecto a las prospectivas de futuro, se parte desde el convencimiento de que esta investigación supone un primer acercamiento a los ámbitos laboral y educacional de los maestros de música castellano-manchegos. Además, hay que considerar que los resultados obtenidos abren nuevos interrogantes que marcan posibles líneas de investigación futuras relacionadas con la formación inicial del profesorado, con la nueva estructura curricular implantada por la LOMCE, con las necesidades formativas del profesorado en activo o con la realización de estudios comparativos entre Comunidades Autónomas que proporcionen una visión general sobre la enseñanza de la música en las escuelas españolas, entre otros asuntos.

Para terminar, es importante reiterar que con este estudio se ha querido dar voz y protagonismo a los responsables directos de la Educación Musical en los colegios de Primaria castellano-manchegos, ya que legislar sin contar con la opinión de los docentes aleja, cada vez más, la norma de la realidad educativa. Solo superando este lastre será más fácil definir currículos de calidad que respeten tanto los procesos de enseñanza-aprendizaje como el trabajo y la identidad de los profesionales.

NARCISO JOSÉ LÓPEZ GARCÍA. THE CONTENT OF THIS ARTICLE IS THE SOLE RESPONSIBILITY OF THE AUTHORS. THE REVISTA ELECTRÓNICA DE LEEME AND UNIVERSITAT DE VALĖNCIA ARE NOT LIABLE FOR ANY LEGAL ACTIONS THAT MAY ARISE INVOLVING THE ARTICLE'S CONTENT. REVISTA ELECTRÓNICA DE LEEME - LISTA ELECTRÓNICA EUROPEA DE MÚSICA EN LA EDUCACIÓN-HTTP://MUSICA.REDIRIS. ES.ISSN: 1575-9563 EDITORES: UNIVERSIDAD DE VALENCIA Y JESÚS TEJADA GIMÉNEZ. VISIBILIDAD DE ESTA REVISTA: EBSCO, CINDOC (CESIC), CITEFACTOR, COPAC, DIALNET, DICE (CSIC), DOAJ, E-REVISTAS (CSIC), EBSCO PREMIER, ERIH+, GALE CENGAGE LEARNING, IN-RECS, IRESIE, LATINDEX, MIAR, OCLC WORLDCAT, RESH, REDIB, RILM CORE JOURNALS, SUDOC, ULRICHS, 


\section{Referencias}

Álvarez, J. L. (2004). Escuela, familia y comunidad educativa. En M. M. Del Pozo (Ed.), Teorías e instituciones contemporáneas de Educación (pp. 131-154). Madrid: Biblioteca Nueva.

Álvarez, C. y San Fabián, J. L. (2012). La elección del estudio de caso en investigación educativa. Gazeta de Antropología, 28 (1). Recuperado de http://www.gazetaantropologia.es/wpcontent/uploads/G28-1-14-CarmenAlvarez-JoseLuisSanFabian.pdf

Andreu, M. y Godall, P. (2012). La importancia de la educación artística en la enseñanza obligatoria: la adquisición de las competencias básicas de Primaria en un centro integrado de música. Revista de Educación, 357, 179-202.

Campoy, T.J. y Gomes, E. (2009). Técnicas e instrumentos cualitativos de recogida de datos. En A. Pantoja (Coord.), Manual básico para la realización de tesinas, tesis y trabajos de investigación (pp. 275-302). Madrid: EOS.

Carbajo, C. (2008). Autopercepción de competencias musicales en el docente de música de primaria. En A. Álamo y M. Luceño (Eds.), Actas del I Congreso sobre Educación e Investigación Musical. Metodologías Aplicadas y Enfoques Pedagógicos en la Enseñanza Musical (pp. 143-151). Madrid: Enclave Creativa.

Carbajo, C. (2009). El perfil profesional del docente de música de educación primaria: autopercepción de competencias profesionales y la práctica de aula. Murcia: Universidad de Murcia.

Cardona, M.C. (2002). Introducción a los métodos de investigación en educación. Madrid: EOS.

Cardona, J. (2008). Cuestiones en torno a la formación y desarrollo profesional de los profesores. Madrid: Sanz y Torres.

Castañer, M. Camerino, O. y Anguera, M. T. (2013). Métodos mixtos en la investigación de las ciencias de la actividad física y el deporte. Apunts. Educación Física y Deportes, 112, 31-36.

Castro, W. F. y Godino, J. D. (2011). Métodos mixtos de investigación en las contribuciones a los simposios de la SEIEM (1997-2010). En M. Marín, G. Fernández, J. Blanco y M.M. Palarea (Coords.), Investigación en Educación Matemática XV (pp. 99-116). Madrid: Sociedad Española de Investigación en Educación Matemática.

Colás, P. y Hernández, G. (2014). Incidencia de la formación del profesorado en sus creencias sobre el valor de las TIC en la enseñanza de la Música. Educatio Siglo XXI, 3 (32), 51-74.

NARCISO JOSÉ LÓPEZ GARCIAA. THE CONTENT OF THIS ARTICLE IS THE SOLE RESPONSIBILITY OF THE AUTHORS. THE REVISTA ELECTRÓNICA DE LEEME AND UNIVERSITAT DE VALÈNCIA ARE NOT LIABLE FOR ANY LEGAL ACTIONS THAT MAY ARISE INVOLVING THE ARTICLE'S CONTENT, REVISTA ELECTRÓNICA DE LEEME - LISTA ELECTRÓNICA EUROPEA DE MÚSICA EN LA EDUCACIÖN-HTTP.//MUSICA.REDIRIS.ES.ISSN: 1575-9563 EDITORES: UNIVERSIDAD DE VALENCIA Y JESÚS TEJADA GIMÉNEZ. VISIBILIDAD DE ESTA REVISTA: EBSCO, CINDOC (CESIC), CITEFACTOR, COPAC, DIALNET, DICE (CSIC), DOAJ, E-REVISTAS (CSIC), EBSCO PREMIER, ERIH+, GALE CENGAGE LEARNING, IN-RECS, IRESIE, LATINDEX, MIAR, OCLC WORLDCAT, RESH, REDIB, RILM CORE JOURNALS, SUDOC, ULRICHS, ZEITSCHRIFTDATENBANK, ESTA REVISTA ESTÁ PUBLICADA CON EL APOYO INSTITUCIONAL DE REDIRIS-CONSEJO SUPERIOR DE INVESTIGACIONES CIENTIFICAS Y ES DE ACCESO LIBRE. 
Comellas, M.J. (2009). Familia y escuela: compartir la educación. Barcelona: Graó.

De Benito, L. A. (2012). ¿Para qué? En A. Álamo y E. Molina (Eds.), Actas del II Congreso CEIMUS de Educación e Investigación Musical. Innovación, motivación y creatividad en la enseñanza musical (pp. 368-373). Madrid: Enclave Creativa.

De Moya, M. V. (2006). Por qué es imprescindible la educación musical. Idea. La Mancha. Revista de Educación de Castilla-La Mancha, 4 (2), 256-259.

De Moya, M. V., Bravo, R. y García, F. J. (2008). Análisis de los estilos de aprendizaje de los estudiantes de magisterio (educación musical). En A. Álamo y M. Luceño (Eds.), Actas del I Congreso sobre Educación e Investigación Musical. Metodologías Aplicadas y Enfoques Pedagógicos en la Enseñanza Musical (pp. 76-88). Madrid: Enclave Creativa.

Domínguez, M. A. (2014). La Educación Musical en el desarrollo de la LOMCE en Aragón. Fórum Aragón, 12, 50-52.

Epelde, A. (2005). Implicaciones de la música en el currículum de educación primaria y en la formación inicial del Maestro especialista de educación musical. Un estudio cualitativocuantitativo en la comunidad andaluza y en las ciudades autónomas de Ceuta y Melilla. Granada: Servicio de Publicaciones de la Universidad de Granada.

Fernández, F. (2008). Obstáculos percibidos por el profesorado de educación musical en los libros de texto. Investigación en la escuela, 65, 49-58.

Gil, F. (1994). Investigando las relaciones familia-escuela: la participación de los padres en el centro. Aula. Revista de Enseñanza e Investigación Educativa, 6, 67-76.

Gil, P., Perona, J. M., Andrés, E. y Hernández, J. R. (2001). Los intereses curriculares de los alumnos/as y de los padres/madres de educación primaria y secundaria obligatoria: especial consideración por el área de educación física. Ensayos, 16, 183-205.

Giráldez, A. (2006). Revisión de la literatura y técnicas de recogida de datos. En M. Díaz Gómez (Coord.), Introducción a la investigación en Educación Musical (pp. 117-155). Madrid: Enclave Creativa.

González, M. y Fuentes, E. J. (2011). El Practicum en el aprendizaje de la profesión docente. Revista de Educación, 354, 47-70.

Guerrero, J. L. (2014). Evaluando actitudes y usos de las TIC de profesorado de música de educación secundaria. Revista Internacional de Educación Musical, 2, 10-23.

Hernández, J. R. (2011). Efectos de la implementación de un programa de educación musical basado en las TIC sobre el aprendizaje de la música en educación primaria. Alicante: Universidad de Alicante.

NARCISO JOSÉ LÓPEZ GARCÍA. THE CONTENT OF THIS ARTICLE IS THE SOLE RESPONSIBILITY OF THE AUTHORS. THE REVISTA ELECTRÓNICA DE LEEME AND UNIVERSITAT DE VALĖNCIA ARE NOT LIABLE FOR ANY LEGAL ACTIONS THAT MAY ARISE INVOLVING THE ARTICLE'S CONTENT. REVISTA ELECTRÓNICA DE LEEME - LISTA ELECTRÓNICA EUROPEA DE MÚSICA EN LA EDUCACIÓN-HTTP://MUSICA.REDIRIS.ES.ISSN: 1575-9563 EDITORES: UNIVERSIDAD DE VALENCIA Y JESÚS TEJADA GIMÉNEZ. VISIBILIDAD DE ESTA REVISTA: EBSCO, CINDOC (CESIC), CITEFACTOR, COPAC, DIALNET, DICE (CSIC), DOAJ, E-REVISTAS (CSIC), EBSCO PREMIER, ERIH+, GALE CENGAGE LEARNING, IN-RECS, IRESIE, LATINDEX, MIAR, OCLC WORLDCAT, RESH, REDIB, RILM CORE JOURNALS, SUDOC, ULRICHS, ZEITSCHRIFTDATENBANK, ESTA REVISTA ESTÁ PUBLICADA CON EL APOYO INSTITUCIONAL DE REDIRIS-CONSEJO SUPERIOR DE INVESTIGACIONES CIENTIFICAS Y ES DE ACCESO LIBRE. 
Latorre, A., Rincón, D. y Arnal, J. (2003). Bases metodológicas de la investigación educativa. Barcelona: Experiencia.

Longueira, S. (2011). Educación musical: un problema emergente de intervención educativa. Indicadores pedagógicos para el desarrollo de competencias en educación musical. Santiago de Compostela: Universidad de Santiago de Compostela.

López García, N. J. (2010). Percepción, conocimiento y opinión de la comunidad educativa sobre la educación musical en primaria. En G. Rusinek, M.E. Riaño y N. Oriol (Eds.), Actas del Seminario Internacional de Investigación en Educación Musical (pp. 170-177). Madrid: Universidad Complutense de Madrid-Sociedad para la Educación Musical del Estado Español.

López García, N. J. (2015). Necesidades profesionales del profesorado especialista de música de los centros de Primaria de Castilla-La Mancha. Málaga: Universidad de Málaga.

Lozano, L. M. y De la Fuente, E. I. (2009). Diseño y validación de cuestionarios. En A. Pantoja (Coord.), Manual básico para la realización de tesinas, tesis y trabajos de investigación (pp. 251-276). Madrid: EOS.

Malbrán, S. (2006). La investigación musical cuantitativa: un recorrido desde la práctica. En M. Díaz Gómez (Coord.), Introducción a la investigación en Educación Musical (pp. 32-59). Madrid: Enclave Creativa.

Martí, L. (2008). Familia y escuela ¿Se invaden, se necesitan...? Cuadernos de Pedagogía, 378, 46-51.

Mominó, J. M., Sigalés, C. y Meneses, J. (2008). La escuela en la sociedad red. Internet en la educación primaria y secundaria. Barcelona: Ariel.

Monarca, H., Fernández-González, N. y Piedrahita, C. (2016). ¿Qué opina el profesorado sobre la LOMCE? Revista Educación, Política y Sociedad, 1 (2), 197-212.

Montesinos, R. M. (2009/2010). El maestro especialista: formación musical inicial y praxis de la educación musical escolar. Tenerife: Servicio de Publicaciones de la Universidad de La Laguna.

Morales, A. (2008). La formación musical en los Maestros para la etapa de educación primaria en la LOGSE y en la LOE. En A. Álamo y M. Luceño (Eds.), Actas del I Congreso sobre Educación e Investigación Musical. Metodologías Aplicadas y Enfoques Pedagógicos en la Enseñanza Musical (pp. 25-38). Madrid: Enclave Creativa.

Ocaña, A. (2006). Identidad y ciclos de desarrollo profesional de los maestros y maestras de educación musical. Granada: Servicio de Publicaciones de la Universidad de Granada.

Okuda, M. y Gómez-Restrepo, C. (2005). Métodos de investigación cualitativa: triangulación. Revista Colombiana de Psiquiatría, 1 (34), 118-124.

NARCISO JOSÉ LÓPEZ GARCÍA. THE CONTENT OF THIS ARTICLE IS THE SOLE RESPONSIBILITY OF THE AUTHORS. THE REVISTA ELECTRÓNICA DE LEEME AND UNIVERSITAT DE VALËNCIA ARE NOT LIABLE FOR ANY LEGAL ACTIONS THAT MAY ARISE INVOLVING THE ARTICLE'S CONTENT. REVISTA ELECTRÓNICA DE LEEME - LISTA ELECTRÓNICA EUROPEA DE MÚSICA EN LA EDUCACIÓN-HTTP://MUSICA.REDIRIS.ES.ISSN: 1575-9563 EDITORES: UNIVERSIDAD DE VALENCIA Y JESÚS TEJADA GIMÉNEZ. VISIBILIDAD DE ESTA REVISTA: EBSCO, CINDOC (CESIC), CITEFACTOR, COPAC, DIALNET, DICE (CSIC), DOAJ, E-REVISTAS (CSIC), EBSCO PREMIER, ERIH+, GALE CENGAGE LEARNING, IN-RECS, IRESIE, LATINDEX, MIAR, OCLC WORLDCAT, RESH, REDIB, RILM CORE JOURNALS, SUDOC, ULRICHS, ZEITSCHRIFTDATENBANK, ESTA REVISTA ESTÁ PUBLICADA CON EL APOYO INSTITUCIONAL DE REDIRISCCONSEJO SUPERIOR DE INVESTIGACIONES CIENTIFICAS Y ES DE ACCESO LIBRE. 
Pastor, P. (2004). Las marías.www.marchitopensil.boe.es. Revista Electrónica de LEEME, 14. Recuperado de http://musica.rediris.es/leeme/revista/pastor04.pdf

Pastor, J. J. (2008). La didáctica de la música: oportunidades para una investigación coordinada. En A. Álamo y M. Luceño (Eds.), Actas del I Congreso sobre Educación e Investigación Musical. Metodologías aplicadas y enfoques pedagógicos en la enseñanza musical (pp. 464472). Madrid: Enclave Creativa.

Pliego, V. (2008). La educación musical en España entre 1988 y 2008 desde una perspectiva periodistica. Madrid: Musicalis.

Reyes, M. L. (2005). La música en educación primaria. Una perspectiva desde el maestro especialista. Granada: Servicio de publicaciones de la Universidad de Granada.

Reyes, M. L. (2010). Evaluación de los planes de estudio de formación del profesorado de Educación Musical: España. Profesorado. Revista de Currículum y Formación del Profesorado, 2 (14), 67-81.

Roche, E. (2010). El secreto es la pasión. Reflexiones sobre educación musical. Barcelona: Clivis.

Rodríguez Fernández, S., Gallardo, M. A., Ruiz, F. y Olmos, M. C. (2005). Investigación educativa: metodología de encuesta. Granada: Grupo Editorial Universitario. Universidad de Granada. Facultad de Educación y Humanidades. Campus de Melilla.

Rodríguez Ruiz, O. (2005). La triangulación como estrategia de investigación en Ciencias Sociales. Revista de Investigación en Gestión de la Innovación y Tecnología, 31. Recuperado de http://www.madrimasd.org/revista/revista31/tribuna/tribuna2.asp

Rosa, F. C. (2015). La formación musical del futuro profesorado de Educación Primaria (Tesis doctoral inédita). Universidad de La Coruña.

Sabariego, M. (2010). Etnografía y estudio de casos. En S. Nieto (Ed.), Principios, métodos y técnicas esenciales para la investigación educativa (pp. 425-445). Madrid: Dykinson.

Salazar, A. (2009). Evolución de la producción de espectáculos de didáctica musical en Andalucía. Situación de su distribución actual y dos propuestas. Papeles del festival de música española de Cádiz. Revista Anual, 4, 123-135.

Sauras, P. (2016). Complejidad de la aplicación de la LOMCE. Revista Educación, Política y Sociedad, 1 (2), 145-162.

Schafer, M. (2008). El rinoceronte en el aula. Buenos Aires: Melos. NARCISO JOSÉ LÓPEZ GARCía. THE CONTENT OF THIS ARTICLE IS THE SOLE RESPONSIBILITY OF THE AUTHORS. THE REVISTA ELECTRÓNICA DE LEEME AND UNIVERSITAT DE
VALĖNCIA ARE NOT LIABLE FOR ANY LEGAL ACTIONS THAT MAY ARISE INVOLVING THE ARTICLE'S CONTENT. REVISTA ELECTRÓNICA DE LEEME - LISTA ELECTRÓNICA EUROPEA DE MÚSICA EN LA EDUCACIÓN-HTTP://MUSICA.REDIRIS.ES.ISSN: 1575-9563 EDITORES: UNIVERSIDAD DE VALENCIA Y JESÚS TEJADA GIMÉNEZ. VISIBILIDAD DE ESTA REVISTA: EBSCO, CINDOC (CESIC), CITEFACTOR, COPAC, DIALNET, DICE (CSIC), DOAJ, E-REVISTAS (CSIC), EBSCO PREMIER, ERIH+, GALE CENGAGE LEARNING, IN-RECS, IRESIE, LATINDEX, MIAR, OCLC WORLDCAT, RESH, REDIB, RILM CORE JOURNALS, SUDOC, ULRICHS, ZEITSCHRIFTDATENBANK, ESTA REVISTA ESTÁ PUBLICADA CON EL APOYO INSTITUCIONAL DE REDIRIS-CONSEJO SUPERIOR DE INVESTIGACIONES CIENTIFICAS Y ES DE ACCESO LIBRE. 
Serrano, J. A., Lera, A. y Contreras, O. (2007). Maestros generalistas vs especialistas. Claves y discrepancias en la reforma de la formación inicial de los Maestros de Primaria. Revista de Educación, 344, 533-555.

Sevillano, M. L. y Fuero, R. (2013). Formación inicial del profesorado en TICS: un análisis de Castilla - La Mancha. Profesorado. Revista de Currículum y Formación del Profesorado, 3 (17), 152-183.

Thode, M. L. (1992). Problemas específicos del colectivo de profesores interinos. Revista Interuniversitaria de Formación del Profesorado, 14, 117-135.

Tiana, A. (1993). Evaluación de centros y evaluación del sistema educativo. Bordón. Revista de Pedagogía, 3 (45), 283-294.

Tójar, J. C. y Matas, A. (2009). Fundamentos metodológicos básicos. En A. Pantoja (Coord.), Manual básico para la realización de tesinas, tesis y trabajos de investigación (pp. 127-152). Madrid: EOS.

Vázquez-Cano, E. (2016). Dificultades del profesorado para planificar, coordinar y evaluar competencias claves. Un análisis desde la Inspección de Educación. Revista Complutense de Educación, 3 (27), 1061-1083.

Vicente, G. (2010). El libro de texto en educación musical. Espiral. Cuadernos del Profesorado, $5(3), 30-35$.

Vilar, M. y Gómez, I. (2005). De la formación a la práctica: metodología para la evaluación de la formación de maestros de música. Revista Interuniversitaria de Formación del Profesorado, $19(2), 175-196$.

NARCISO JOSÉ LÓPEZ GARCÍA. THE CONTENT OF THIS ARTICLE IS THE SOLE RESPONSIBILITY OF THE AUTHORS. THE REVISTA ELECTRÓNICA DE LEEME AND UNIVERSITAT DE VALËNCIA ARE NOT LABE FOR ANY LEGA ACTIONS THAT MAY ARISE INVOVING THE ARTICLE'S CONTENT. REVISTA ELECTRÓNCA DE LEEME - LISTA ELECTRÓNICA EUROPEA DE MÚSICA EN LA EDUCACIÓN-HTTP://MUSICA.REDIRIS. ES.ISSN: 1575-9563 EDITORES: UNIVERSIDAD DE VALENCIA Y JESÚS TEJADA GIMÉNEZ. VISIBILIDAD DE ESTA REVISTA: EBSCO, CINDOC (CESIC), CITEFACTOR, COPAC, DIALNET, DICE (CSIC), DOAJ, E-REVISTAS (CSIC), EBSCO PREMIER, ERIH+, GALE CENGAGE LEARNING, IN-RECS, IRESIE, LATINDEX, MIAR, OCLC WORLDCAT, RESH, REDIB, RILM CORE JOURNALS, SUDOC, ULRICHS, ZEITSCHRIFTDATENBANK, ESTA REVISTA ESTÁ PUBLICADA CON EL APOYO INSTITUCIONAL DE REDIRIS-CONSEJO SUPERIOR DE INVESTIGACIONES CIENTIFICAS Y ES DE ACCESO LIBRE. 